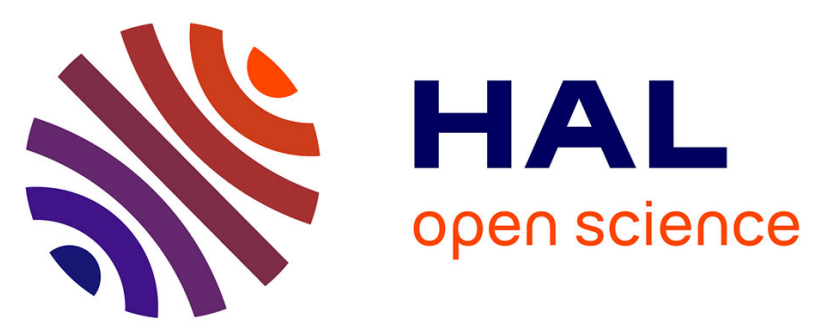

\title{
Inverse ray tracing on icosahedral tetrahedron grids for non-linear laser plasma interaction coupled to 3D radiation hydrodynamics
}

\author{
A. Colaïtis, I. Igumenshchev, J. Mathiaud, V. Goncharov
}

\section{- To cite this version:}

A. Colaïtis, I. Igumenshchev, J. Mathiaud, V. Goncharov. Inverse ray tracing on icosahedral tetrahedron grids for non-linear laser plasma interaction coupled to 3D radiation hydrodynamics. Journal of Computational Physics, 2021, 443, pp.110537. 10.1016/j.jcp.2021.110537 . hal-03377152

\author{
HAL Id: hal-03377152 \\ https://hal.science/hal-03377152
}

Submitted on 14 Oct 2021

HAL is a multi-disciplinary open access archive for the deposit and dissemination of scientific research documents, whether they are published or not. The documents may come from teaching and research institutions in France or abroad, or from public or private research centers.
L'archive ouverte pluridisciplinaire HAL, est destinée au dépôt et à la diffusion de documents scientifiques de niveau recherche, publiés ou non, émanant des établissements d'enseignement et de recherche français ou étrangers, des laboratoires publics ou privés. 


\title{
Inverse ray tracing on icosahedral tetrahedron grids for non-linear laser plasma interaction coupled to 3D radiation hydrodynamics
}

\author{
A. Colaïtis ${ }^{\mathrm{a}}$, I. Igumenshchev ${ }^{\mathrm{b}}$, J. Mathiaud ${ }^{\mathrm{a}}$, V. Goncharov ${ }^{\mathrm{b}}$ \\ ${ }^{a}$ Centre Lasers Intenses et Applications, UMR 5107, 351 Cours de la libération, 33400 Talence, France \\ ${ }^{b}$ Laboratory for Laser Energetics, 250 East River Rd, Rochester, NY 14623-1212
}

\begin{abstract}
A novel approach to efficiently model 3-D laser plasma interactions at fluid scales is presented. This method, implemented in the IFRIIT propagation code developed at CELIA, relies on inverse ray tracing to compute laser fields at arbitrary locations in a plasma. This enables to describe the fields at high order in space compared to standard forward ray tracing approaches. In addition, inverse ray tracing enables the use of etalon integral methods to reconstruct caustic fields and greatly speeds up calculations of cross-beam energy transfer by decoupling the ray amplitude and ray phase calculations. A comparison of the inverse and forward methods for 3-D calculations of fields in presence or not of cross-beam energy transfer illustrates the significant advantages of the inverse method. Conversely, while the inverse method is well suited to most spherical plasma profiles, it currently cannot treat concave profiles or target holders. The coupling of IFRIIT with the 3-D ASTER radiative hydrodynamics code developped at the Laboratory for Laser Energetics is then presented. ASTER and IFRIIT resolve their respective equations on separate grids which communicate through interpolation. As such, IFRIIT uses a dedicated laser grid adapted to the computations at play, which also allows to use different parallelization methods for both codes: block decomposition for the hydrodynamics versus domain duplication for the laser. Applications to direct-drive implosions for inertial confinement fusion are presented, for which a geodesic icosahedron grid is implemented in IFRIIT. The performances of the ASTER/IFRIIT coupling is demonstrated by conducting simulations of cryogenic implosions performed on the OMEGA laser system, in presence of various sources of 3-D effects; laser port geometry, cross-beam energy transfer, beam imbalance and target mis-alignment. Comparison with neutron data, measured through bang-time, for a cryogenic implosion experiment shows an excellent agreement for the laser-plasma coupling.
\end{abstract}

Keywords:

\section{Introduction}

Three major avenues to Inertial Confinement Fusion (ICF) are currently being explored worldwide [1]: Laser Direct-Drive (LDD) [2], Laser (x-ray) Indirect-Drive (LID) [3] and magnetic drive using pulsed power [4]. These approaches have in common the use of laser beams and face challenges related to Laser Plasma Instabilities (LPI). LPIs are non-linear microscopic processes which couple plasma eigen-modes (electron or ion plasma waves) to either the laser beams, scattered light, or with each other [5]. In the case of LDD and LID, the main LPIs at play are CrossBeam Energy Transfer (CBET), Stimulated Raman Scattering (SRS), Stimulated Brillouin Scattering (SBS) and TwoPlasmon Decay (TPD). Their consequences on large-scale plasma hydrodynamics are often important, leading to significant loss of laser/target coupling, introducing asymmetries in target compression, and generating supra-thermal electron populations [6].

While LPIs have a paramount influence in ICF, they are also highly difficult to model in Radiative Hydrodynamics (RH) codes that describe the plasma motion at fluid scales. This relates to an incompatibility of dimensions between the typical plasma size $(\sim \mathrm{mm})$ and driver duration $(\sim \mathrm{ns})$ compared to the scales required to resolve the kinetic processes at play in LPIs $(\sim \mathrm{nm}$ and $\sim \mathrm{fs})$. These 6 orders of magnitudes differences in time and space prevent direct numerical calculations of LPIs in 3-D geometries and at fluid scales. As such, they are often computed at reduced scales, for short period of times, in reduced number of dimensions and/or for limited number of laser beams. For these 
reasons, models for laser propagation in hydrodynamics codes have long been limited to the linear process of collisional absorption. This is usually modeled in the Geometrical Optics (GO) framework for computing laser trajectories [7], which offers adequate performances at fluid scales and even in 3-D geometries.

Given the importance of LPIs in ICF, significant efforts have been made in the last 10 to 15 years to include reduced LPI models in fluid codes, mainly based on GO models for numerical efficiency reasons. These reduced models must address two main questions: (i) how to compute laser intensities or fields in the GO framework, key properties for LPIs and (ii) how to account for the microscopic processes. The first issue stems from the infinitely thin property of GO rays, which prevents a straightforward definition of a ray intensity. The GO framework also breaks down at laser caustics, which are prominent in LDD. The second issue relates to the formulation of theoretical models that reproduce microscopic physics from macroscopic quantities. Such models have notably been proposed for CBET by considering the laser wave as locally plane and homogeneous $[8,9,10]$, and are used across a variety of codes. However, significant technical difficulties arise in coupling these models with the intensity calculation methods of GO. Usually, such details are eluded with the introduction of free numerical parameters in CBET models, thus allowing to tune results of calculations to match experimental data. While some of these parameters simply limit the amplitude of calculated ion waves to physical values, others may directly tune the caustic fields to unphysical values simply to set the desired amount of CBET. In this paper, we aim at proposing a fluid-scale laser model, IFRIIT, which follows consistently the GO framework to compute laser fields and that eliminates such free parameters related to caustic fields, thus improving model reliability and scope of application for those cases where some non-linear couplings occur at caustics (as is the case in direct-drive, see an illustration in Sec. 5.2.2). In addition, we present the coupling of IFRIIT to the ASTER [11, 12] RH code for the specific spherical geometries of LDD. Calculations presented in the paper are conducted in the framework of the OMEGA laser system configuration and diagnostics [13].

Our algorithm differs significantly from other methods implemented in RH codes on several key points. First, we make use of Inverse Ray Tracing (IRT) to compute the laser propagation, by opposition with the method of Forward Ray Tracing (FRT) that is usually employed. That is the first time an IRT method is used for laser calculations coupled to plasma hydrodynamics in RH fusion codes. Second, we decouple the laser grid from the hydrodynamics grid. This allows to tailor a grid that is optimized for the resolution of the ray equations, leading to better load balance for parallel computations. In addition, the laser grid itself is split in two grids; one for calculation of ray electric fields, and one for calculation of ray trajectories. Furthermore, we employ a geodesic laser grid structure, contrary to the standard polar grids employed in 3-D RH codes. It is the first time that such a three-grids scheme is employed for laser calculations. Third, we make use of the Etalon Integral method to compute laser fields in places where the GO framework breaks down. This is also a new technique for RH codes, which is enabled by the use of IRT and allows to remove the free parameters used in standard codes to set caustic fields. Fourth, the fields reconstructed from GO using IRT are done so at higher order in space than with FRT. This is also the first time that such higher order field estimations are made possible in RH codes. This is an important point in 3-D ICF implosion, where noise issues stemming from FRT are often detrimental. Fifth, the laser code and the RH codes are also decoupled in time, allowing both codes to iterate asynchronously. Indeed, laser computations are often more costly than hydrodynamic ones (when including LPIs), but do not require to be updated as often. This is also the first time a RH code implements a desynchronized laser scheme. Last, as will be shown in the paper, the use of IRT has additional advantages; e.g. it allows a proper separation of the laser field between the various reflected components (so-called sheets), which, for example, enables to precisely account for self interaction of the incident and reflected fields of beams through CBET. It also allows to considerably speed up pump depletion iterations in CBET algorithms, by allowing to update only parts of the ray equations.

This paper documents the details of the IFRIIT model, its coupling to the ASTER code, and illustrates the code results and performance in numerical tests and typical LDD applications. First, the mathematical formulation of the GO framework is recalled in Sec. 2.1. Here, IFRIIT uses the ray field formulation, as opposed to the ray power formulation used in most RH codes. From this we introduce the notion of caustics and ray sheets in Secs. 2.2 and 2.3, and detail all the physics models used in IFRIIT in Sec. 2.4. Following these first definitions, we discuss the various methods for computing laser propagation in current RH codes in Sec. 3.1. The IFRIIT grid model and IRT method are then detailed in Secs. 3.2 and 3.3, with limitations of the current approach discussed in 3.4. The coupling of IFRIIT to ASTER in the three-grid approach is described in Sec. 4. This includes a description of the various grids, interpolation methods, heterogeneous parallelization scheme, asynchronous laser/hydrodynamics iteration, and the warmup model used for the early interaction. Finally, numerical experiments and applications are presented in Sec. 5, where we compare IRT vs FRT in test cases and typical implosions, as well as discuss various 3-D effects modeled 
by ASTER and IFRIIT, such as laser chamber geometry, CBET, target offset and beam imbalance. Our conclusions are presented in Sec. 6.

\section{Mathematical models for fields}

\subsection{Basic ray equations}

We are interested in describing the propagation of laser electric fields in plasmas at the large scale of high energy density laser experiments. At the most detailed level, this propagation is described by Maxwell's equations, relating electric fields to the dielectric properties $\epsilon$ of the medium. Generally, the plasma may be anisotropic ( $\epsilon$ is a tensor), inhomogeneous ( $\epsilon$ varies in space) and absorptive or dispersive ( $\epsilon$ is frequency dependent and complex). In order to simplify the propagation problem, a few assumptions are made: the laser light is monochromatic of frequency $\omega \equiv \omega_{0}$, the component of the permittivity responsible for trajectory is isotropic, the laser propagation is steady state, and the electric field amplitude varies slowly in space compared to the wavelength of the light. These simplifications allow to seek solutions for the field from the scalar Helmholtz equation [14]:

$$
\Delta u(\mathbf{r})+k_{0}^{2} \epsilon(\mathbf{r}) u(\mathbf{r})=0
$$

where $\mathbf{r}$ is a spatial coordinate, $k_{0}=2 \pi / \omega_{0}$ is the vacuum wavevector norm with $\omega_{0}$ the vacuum laser frequency, $\epsilon$ is the complex-valued permittivity of the medium, $u$ is the laser field defined in units of the normalized oscillation velocity, i.e. $u=v_{\mathrm{osc}} / c=e E /\left(m_{e} \omega_{0} c\right)$, with $E$ the electric field in SI units, $e$ the electron charge, $m_{e}$ the electron mass and $c$ the speed of light, and we have made the further assumption of $s$-polarized light (i.e. $\mathbf{u} . \nabla \epsilon=0$ ). We then seek solutions of this equation in the form of so-called rays, which ansatz reads [14]:

$$
u(\mathbf{r})=A(\mathbf{r}) \exp \left[\imath k_{0} \psi(\mathbf{r})\right],
$$

with $A$ the ray amplitude and $\psi$ the ray phase. This is the Geometrical Optics (GO) framework. Finally, we consider real-valued rays, which stems from the assumption of $\operatorname{Re}(\epsilon) \gg \operatorname{Im}(\epsilon)$ such that the phase is written as $\psi=\psi^{\prime}+\imath \psi^{\prime \prime}$ with $\left|\psi^{\prime}\right| \gg\left|\psi^{\prime \prime}\right|$. In this framework, $A$ is also real-valued because the initial condition of $A(0)$ is, and the field at a given point is $|u|=|A(\mathbf{r})| \exp \left[-k_{0} \psi^{\prime \prime}(\mathbf{r})\right]$. In practice, real-valued GO is valid away from ray turning points (also called caustics, see Sec. 2.3) and for warm plasmas (above a few hundred electron-volts) [15]. It also neglects any diffraction phenomena and as such is only valid on scalelengths shorter that the beam Rayleigh range. For ICF beams, the latter is usually much longer than the typical scale on which LPIs take place.

Rays obey the eikonal and transport equations, derived by injecting the ray field ansatz into the Helmholtz equation and identifying the terms at order 0 and 1 in inverse powers of $\imath k_{0}$, separating real and imaginary parts:

$$
\begin{array}{r}
\left(\nabla \psi^{\prime}\right)^{2}=\epsilon^{\prime}(\mathbf{r}), \\
2 \nabla \psi^{\prime} . \nabla \psi^{\prime \prime}=\epsilon^{\prime \prime}(\mathbf{r}), \\
2(\nabla A . \nabla \psi)+A \Delta \psi=0,
\end{array}
$$

where $\epsilon^{\prime}$ and $\epsilon^{\prime \prime}$ denote the real and imaginary part of the permittivity, respectively.

The eikonal equation (Eq. 3) is solved using the characteristics method to obtain the ray trajectory equations:

$$
\begin{array}{r}
\frac{\mathrm{d} \mathbf{r}}{\mathrm{d} \tau}=\mathbf{p}, \\
\frac{\mathrm{d} \mathbf{p}}{\mathrm{d} \tau}=\frac{1}{2} \nabla \epsilon^{\prime}(\mathbf{r}),
\end{array}
$$

with $\mathbf{r}$ the ray position, $\mathbf{p}$ the ray momentum, and $\tau$ a parametric coordinate related to the ray arc-length $s$ by the differential relation $\mathrm{d} \tau=\mathrm{d} s / \sqrt{\epsilon^{\prime}}$. These equations show that in the real-valued GO approximation, the ray trajectory only depend on $\epsilon^{\prime}$.

Rays are launched at $\tau=0$ from a two-dimensional initial ray surface parametrized by coordinates $\zeta_{1}, \zeta_{2}$ where the boundary condition for the ray field $u\left(\tau=0, \zeta_{1}, \zeta_{2}\right)$ is known. In practice, $\left(\tau=0, \zeta_{1}, \zeta_{2}\right)$ can be seen as a lens plane for the laser wave. The coordinates $\tau, \zeta_{1}, \zeta_{2}$ are called the ray phase space parameters. 
Along the ray trajectory, the field is obtained by resolving for the amplitude $A$ and the phase $\psi^{\prime \prime}$. From Eq. (4) one can obtain:

$$
\frac{\mathrm{d} \psi^{\prime \prime}}{\mathrm{d} \tau}=\frac{\epsilon^{\prime \prime}(\mathbf{r})}{2},
$$

which represents the field damping or amplification due to the medium. This contribution is obtained by integrating $\epsilon^{\prime \prime}$ along the trajectory of the ray (given by Eqs. (6)).

The transport equation (Eq. (5)) can be integrated to give the ray amplitude equation:

$$
A(\tau)=A(0)\left|\frac{D(0)}{D(\tau)}\right|^{1 / 2}, D(\tau)=\left|\begin{array}{lll}
\frac{\partial x}{\partial \zeta_{1}} & \frac{\partial x}{\partial \zeta_{2}} & p_{x} \\
\frac{\partial y}{\partial \zeta_{1}} & \frac{\partial y}{\partial \zeta_{2}} & p_{y} \\
\frac{\partial z}{\partial \zeta_{1}} & \frac{\partial z}{\partial \zeta_{2}} & p_{z}
\end{array}\right|,
$$

where $D$ is the determinant of the Jacobian of the coordinate change from ray phase space to real space and we have used $\partial \mathbf{r} / \partial \tau=\mathbf{p}$. This equation describes conservation of energy in a ray tube; a volume defined by ray trajectories infinitesimally close to each other. When adjacent rays get closer to each other, $A$ increases, and inversely when rays go further away from each other.

In most laser models implemented in RH codes, the laser plasma coupling is described from the ray power and not the ray field. The latter is obtained by integrating the squared field over an infinitesimal cross-section, giving the power conservation equation for the ray:

$$
\frac{\mathrm{d} P}{\mathrm{~d} \tau}=-\epsilon^{\prime \prime} P(\mathbf{r}(\tau))
$$

where $P$ is the ray power.

Note that in that case, the amplitude term of Eq. (8) is no longer present. That is because conceptually, when working with ray power directly, the ray has no physical thickness. The effect of field amplification due to rays bunching up is then obtained only when binning adjacent ray trajectories in cells, as explained in Sec. 5.1, and hence depends on the ray statistics.

\subsection{Ray sheets}

In order to describe the ray amplitude continuously across space, one must be able to evaluate the derivatives in $D$ at all points. This in turn requires to know the mapping $\mathcal{M}$ between phase coordinates $\left(\tau, \zeta_{1}, \zeta_{2}\right)$ and real coordinates $(x, y, z)$. This mapping is decomposed in sub-mappings, called sheets, which are individually bijective with respect to the area lit by the laser. For example, the trajectory of a ray from $\mathbf{r}_{0}=0$ with an initial momentum $\left|\mathbf{p}_{0}\right|=1$ along the $\mathbf{x}$ base vector and onto a constant density gradient $\mathbf{x} . \nabla \epsilon^{\prime}$ is $x(\tau)=\tau\left(1+\tau \mathbf{x} . \nabla \epsilon^{\prime} / 4\right)$ with $y(\tau)=y_{0}$ and $z(\tau)=z_{0}$. In this simple example, we see that there are two positive solutions for $\tau$ in the equation $\hat{x}=x(\tau)\left(\operatorname{assuming} \hat{x}>-1 / \mathbf{x}\right.$. $\nabla \epsilon^{\prime}$ and $\left.\mathbf{x} . \nabla \epsilon^{\prime}<0\right)$. These two solutions correspond to the two bijective sheets: the incident and reflected field.

In the general case, while each sheet is a bijective mapping, the set of sheets $\mathcal{M}$ is not bijective itself. In practice, this means that several ray phase space parameters can lead to rays ending up at the same physical location. This is the case of the example given earlier. Phase space parameters where the sheets join are called caustics. They correspond to locations in real space where the number of solutions to the inverse mapping $(x, y, z) \rightarrow\left(\zeta_{1}, \zeta_{2}, \tau\right)$ changes. At these degeneracy points, the Jacobian of $\mathcal{M}$ is no longer invertible since its determinant is zero, leading to $A \rightarrow \infty$. Using the practical example of earlier, one can find $D(\tau)=\left(1+\tau \mathbf{x} . \nabla \epsilon^{\prime} / 2\right)$ for a plane-wave-like initial condition of $y_{0}=\zeta_{1}$ and $z_{0}=\zeta_{2}$. Here, $D=0$ for $\tau=-2 / \mathbf{x}$. $\nabla \epsilon^{\prime}$, which corresponds to $x=-1 / \mathbf{x}$. $\nabla \epsilon^{\prime}$, where $x(\tau)$ goes from having two solutions to one solution, i.e. when the ray is reflected.

A second example of ray optics in the framework of ray phase space parameters and IRT is given in Fig. 1. Here, we consider a plane wave with super-gaussian profile incident at a $20^{\circ}$ angle with respect to a linear density profile. The first and second sheets are illustrated in real space in Fig. 1 (a,b,c) and in phase space in (d,e,f). The construction of the mapping $\mathcal{M}$ through IRT is illustrated in Fig. $1(\mathrm{~d}, \mathrm{e})$. The Jacobian of the transformation associated with the coordinate mapping in Fig. $1(\mathrm{~d}, \mathrm{e})$ is used to compute the field swelling term $D$ in Eq. 8.

The concept of ray sheets is generic to geometrical optics, but is made straightforward from the mapping of phase space to real space parameters in the framework of the IRT approach. While the latter can be applied in principle to generic plasma profiles, parctical limitations in IRT algorithms convergence currently restrict us to the case of 2 sheets 

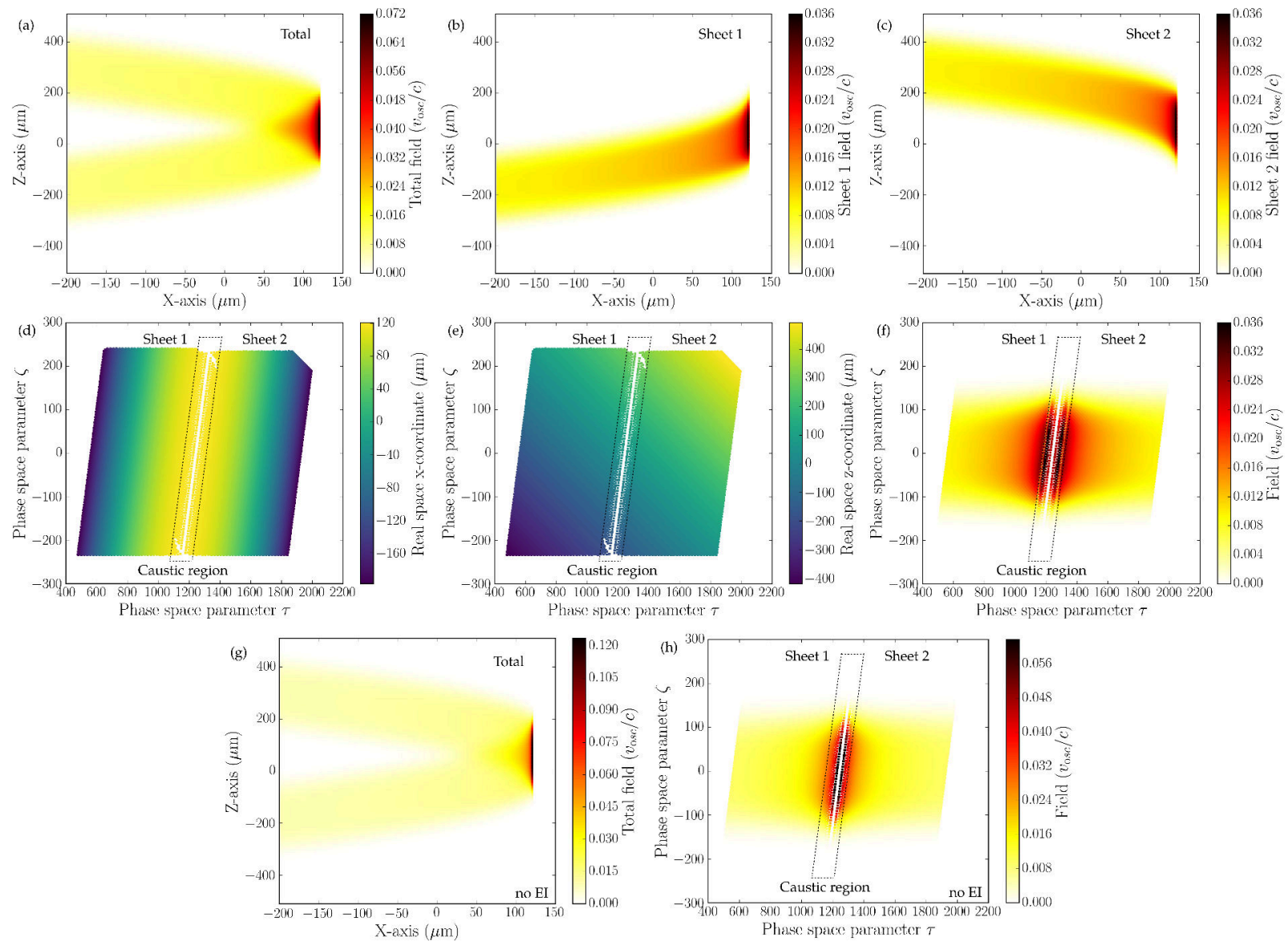

Figure 1: Slice of a beam propagating at a $20^{\circ}$ angle in a $2 \mathrm{D}$ linear permittivity profile in the $X-Z$ plane. The profile has a $365 \mu \mathrm{m}$ scalelength for the real part of the permittivity and the imaginary part is set to 0 - thus removing laser absorption. The beam profile is a $100 \mu \mathrm{m} 1 / \mathrm{e}$ radius super-gaussian of order 4 , and its peak intensity is arbitrarily set to $10^{15} \mathrm{~W} / \mathrm{cm}^{2}$. There is no frequency shift or CBET included in the calculation. Panels (a,b,c) show the beam propagation in real space, with: (a) the total field, (b) the sheet 1 (incident) field, and (c) the sheet 2 (reflected) field. Panels (d,e,f) show the beam in phase space. The mapping from space phase space to real space is given in (d) for coordinate $X$ and (e) for coordinate $Z$. Corresponding fields in phase space are given in (f). The caustic zone, where ray optics is inapplicable, is highlighted as a dashed box in $(\mathrm{d}, \mathrm{e}, \mathrm{f})$. Fields in the case of ray optics without the Etalon Integral method are shown in panels $(\mathrm{g})$ and $(\mathrm{h})$ in real space and phase space, respectively. Note the different color-scale on $(\mathrm{g}, \mathrm{h})$ compared to $(\mathrm{a}, \mathrm{f})$. 
separated by a single caustic of Fold type. This approach is compatible with the framework of many simulations of interest in direct-drive inertial fusion. Similarly to the 2D planar example, we provide a 3D illustration of the mapping for a beam at normal incidence onto a spherical capsule profile in Fig. 2. The plasma and beam configuration is the same that is used and described in Sec. 5.2.1. Figs. 2 (a,b) illustrate the values of the parametric arclength $\tau$ in the incident and reflected fields, respectively, mapped to the lit area of the laser in real space. Notably, one can see in Fig. 2 (a) the initially flat phase front of the laser wave, which deforms as it gets closer to the caustic. In Fig. 2 (b), the phase fronts are now spherically divergent; the wavefield tends toward a spherical wave due to the reflection onto the spherical critical density. In both cases, one can see that the mapping covers a significant portion of the 4 pi of the simulation domain, with a shadow left behind the position of the critical density surface; this is the shadow region. The caustic is located at this lit/shadow interface, and separates the two sheets. One can also note that the values for $\tau$ between sheets 1 and 2 converge at the caustic, as expected. Figures 2 (c,d,e,f) are the equivalent for the other two parameters $\zeta_{1}$ and $\zeta_{2}$. This is easiest to understand in the first sheet, e.g. panel (c); since there is weak refraction far from the critical density, the $\zeta_{1}$ isocontours are almost flat and simply reflect that rays travel mostly straight from the lens plane, thus mapping almost directly from the lens plane to real space.

\subsection{Caustics}

At locations where $D=0$, the GO method is no longer applicable. In practice, the non-applicability domain extends in a so-called caustic region where the phase difference between rays leading to a given point is small [14]. In this region, $D \rightarrow 0$ such that the field value does not diverge to infinity but can take arbitrarily large values. The caustic region is highlighted in Fig. 1, and corresponds to a small region where the sheets are joining up in phase space $(D=0$ exactly at the junction).

In RH codes using the ray field approach, avoiding the divergence at caustics is usually done through a numerical clamping parameter. Other methods (notably in offline codes), usually employ a local maximum field value based on the field expected in a fully linear density profile [16]. In RH codes that infer fields from absorption using Eq. (9), the fields are non-divergent at caustics because there is no swelling term. However, the caustic field depends entirely on the meshing resolution and its value does not converge toward the physical value because GO remains invalid. A further discussion of the various methods employed in RH codes is given in Sec. 3.1.

In IFRIIT, caustic fields are obtained using an Etalon Integral (EI) method, made possible by the use of IRT which allows to know the ray parameters at discrete points in space. This is reported in details in Ref. [15]. The principle of the EI method implemented in IFRIIT is briefly recalled here. We make the assumption that caustics encountered in simulations involve only two rays, i.e. they are of fold type. The field near the fold-type caustic is approximated by the sum of (i) an Airy function, analytical solution for the field in a constant density gradient, and (ii) the derivative of an Airy function, which can account, e.g., for weak caustic curvature or wavefront distortions [14]. The caustic field then takes the form:

$$
u(\mathbf{r}) \simeq\left\{C_{1}(\mathbf{r}) \tilde{I}[\xi(\mathbf{r})]-{ }_{l} C_{2}(\mathbf{r}) \tilde{I}[\xi(\mathbf{r})] / k_{0}\right\} \exp \imath k_{0} \chi(\mathbf{r}),
$$

where $\tilde{I}(\xi)=k_{0}^{1 / 6} \sqrt{2 \pi} \mathrm{Ai}\left(k_{0}^{2 / 3} \xi\right)$ is the dimensional Airy integral and Ai is the Airy function. The four functions of space $C_{1}(\mathbf{r}), C_{2}(\mathbf{r}), \chi(\mathbf{r})$ and $\xi(\mathbf{r})$ introduced in Eq. (10) are then related to the four ray parameters $\left(\psi_{1}, \psi_{2}, A_{1}, A_{2}\right)$ of the two rays 1 and 2 reaching an observation point lying in the caustic region. By matching the fields asymptotically and equating the pre-exponential factors, the caustic field reads:

$$
u=\sqrt{\pi}\left[(-\xi)^{1 / 4}\left(A_{1}+\imath A_{2}\right) \operatorname{Ai}(\xi)-(-\xi)^{-1 / 4}\left(A_{2}+\imath A_{1}\right) \operatorname{Ai}^{\prime}(\xi)\right] \exp \left[\imath\left(k_{0} \chi-\pi / 4\right)\right],
$$

where the $\xi$ and $\chi$ parameters are related to the eikonal of the rays through:

$$
\begin{array}{r}
\chi=\frac{1}{2}\left(\psi_{1}+\psi_{2}\right), \\
\xi=-\left(k_{0} \frac{3}{4}\left(\psi_{2}-\psi_{1}\right)\right)^{2 / 3},
\end{array}
$$

where rays 1 and 2 must be ordered such that $\operatorname{Re}\left(\psi_{1}\right)<\operatorname{Re}\left(\psi_{2}\right)$. 
(a)

(c)
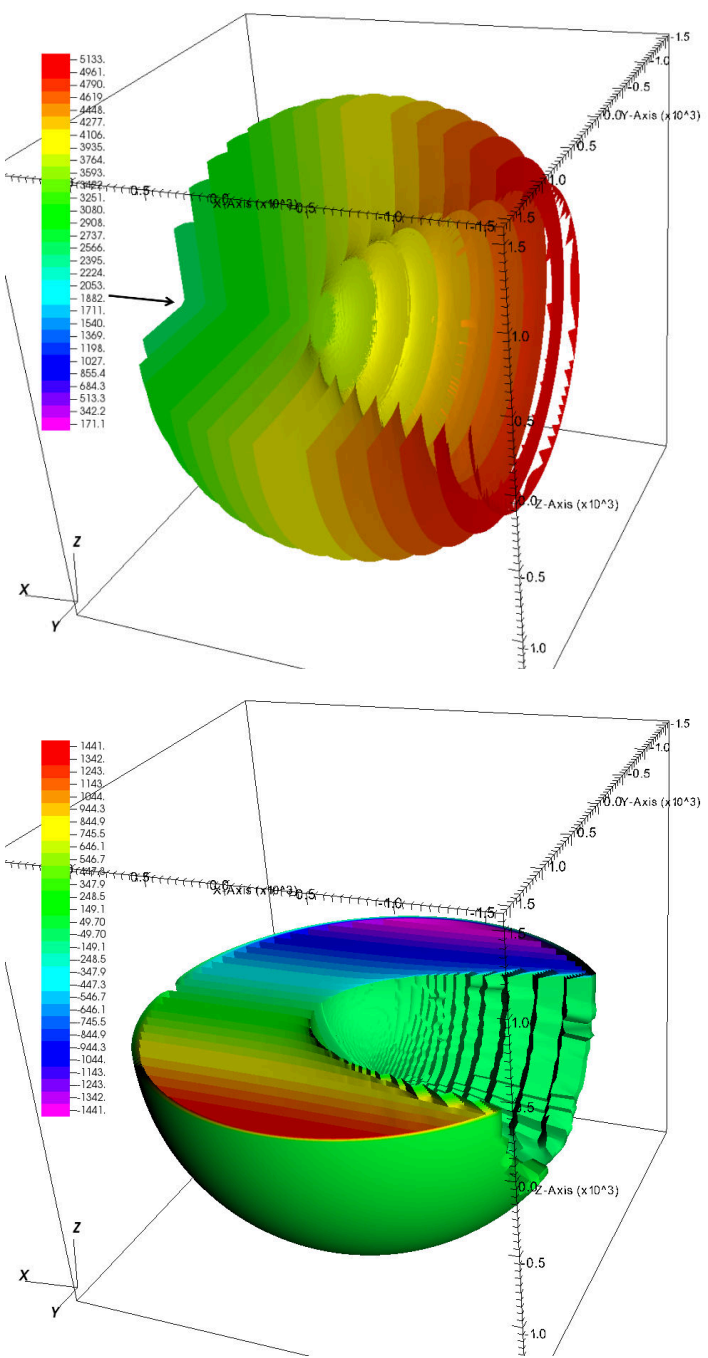

(e)

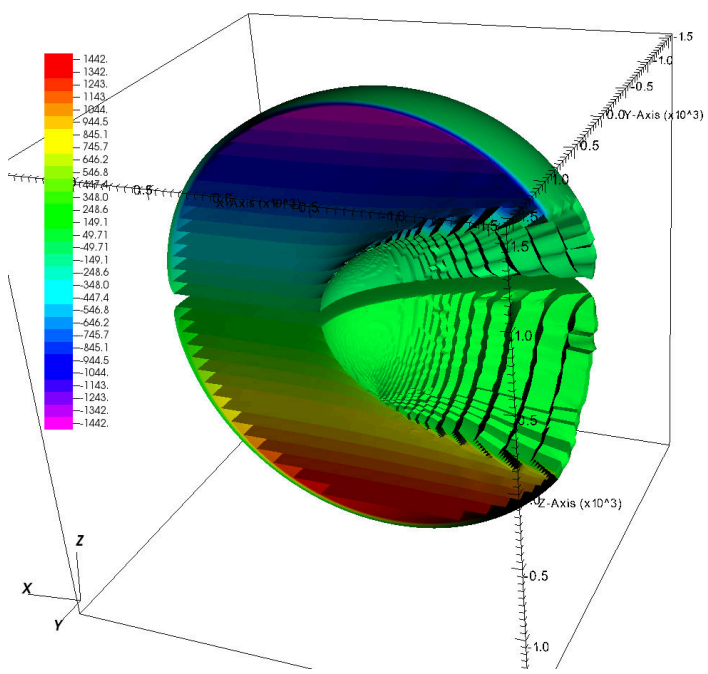

(b)

(d)
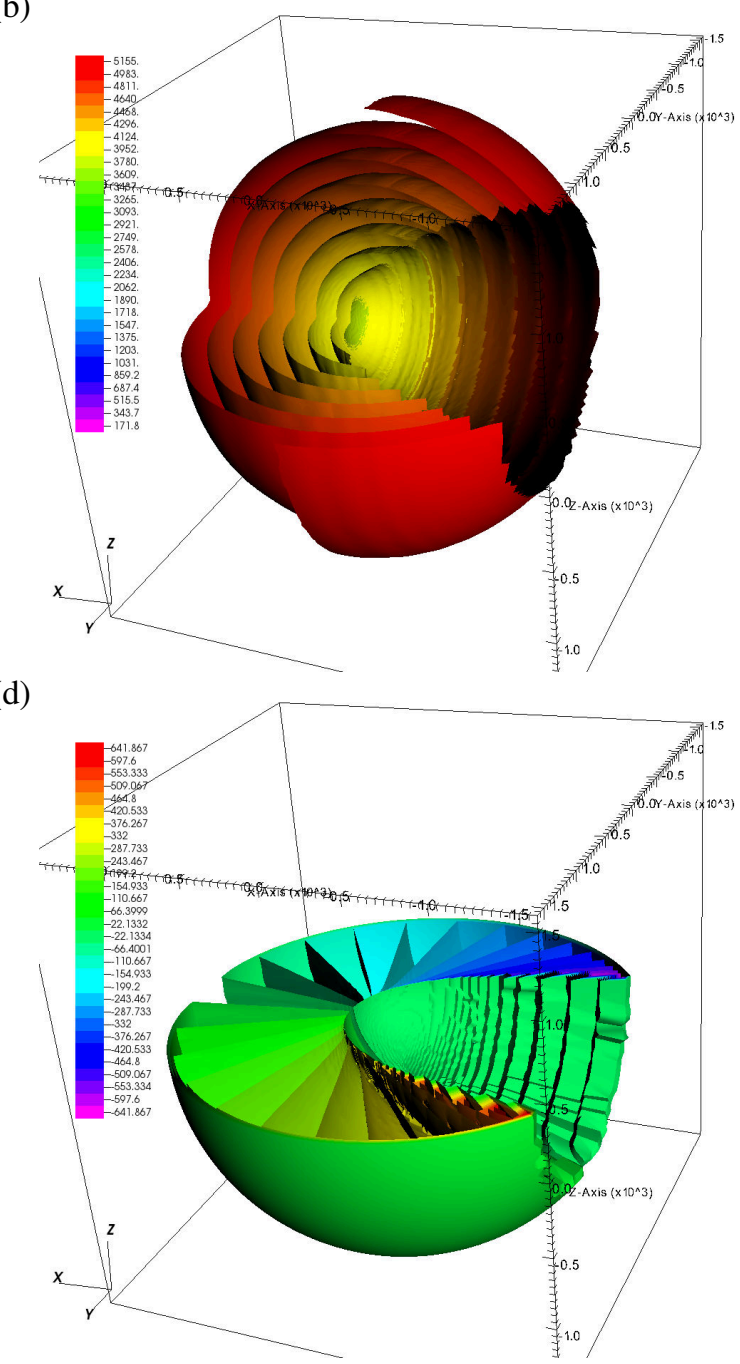

(f)

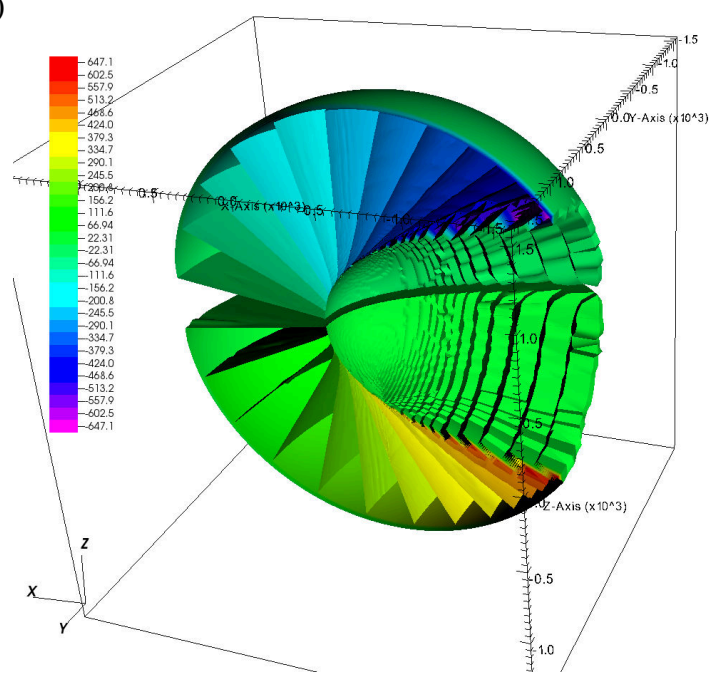

Figure 2: Phase space to real space mapping configuration for a single beam at normal incidence onto a spherical plasma profile relevant to an ICF implosion (see configuration presented in Sec. 5.2.1). The beam is incident from the left (see the arrow in panel (a)). Values are shown as isocontours, for the incident (sheet 1) field on the left column (a,c,e) and for the reflected (sheet 2) field on the right column (b,d,f). The phase space parameters are: the parametric arclength $\tau(\mathrm{a}, \mathrm{b})$, the coordinates $\mathrm{ohto}_{\mathrm{t}}$ the first (c,d) and second (e,f) axises of the initial ray surface, $\zeta_{1}$ and $\zeta_{2}$, respectively. As a reminder, the initial ray surface can be here viewed as a lens plane, but closer to the simulation domain. All mapping values are in units of microns. 


\subsection{Phase integration and the permittivity function}

In addition to the amplitude $A$, the ray field $|u|$ depends on the integral along the ray trajectory of $\epsilon^{\prime \prime}$ (see Eq. (7)). In the IFRIIT model, the imaginary part of the permittivity $\epsilon^{\prime \prime}$ is beam and sheet-dependent. It is decomposed such that the imaginary part of the permittivity seen by beam $i$ in sheet $j$ is:

$$
\epsilon_{i, j}^{\prime \prime}=\epsilon_{0, i}^{\prime \prime} f_{L}+\delta \epsilon_{i, j}^{\prime \prime}
$$

where $\epsilon_{0, i}^{\prime \prime}$ represents the absorption contribution from the plasma due to the inverse Bremsstrahlung (IB) process, $f_{L}$ is an absorption reduction factor that depends on the squared fields from all beams and sheets (see Sec. 2.4.1), and $\delta \epsilon_{i, j}^{\prime \prime}$ is a Cross-Beam Energy Transfer (CBET) exchange term that depends on the fields, k-vector and frequencies of all sheets and beams except $\{i, j\}$ (see Sec. 2.4.2). While $\epsilon_{0, i}^{\prime \prime}$ is straightforward to compute in warm plasmas, the other contributions depend on the other beams, and as such require iterations to compute, as explicited in Sec. 2.4.2.

\subsubsection{Langdon effect on absorption}

The absorption of laser energy by IB occurs preferentially on low-energy electrons, such that the electron distribution function (EDF) becomes distorted from a Maxwellian towards a super-Gaussian function of order $m=5$ [17]. The EDF then relaxes towards a Maxwellian through electron-electron collisions. The amplitude of this effect can be characterized from the ratio $\alpha$ of the IB and e-e frequencies:

$$
\alpha=\frac{Z_{\mathrm{eff}} c^{2}}{v_{\mathrm{th}}^{2}} \sum_{\substack{i \text { beams } \\ j \in \text { sheets }}}\left|u_{i, j}\right|^{2}
$$

with $Z_{\text {eff }}=\frac{\left\langle Z^{2}>\right.}{\langle Z>}$ the effective ion charge and $v_{\text {th }}=\sqrt{k_{\mathrm{B}} T_{e} / m_{e}}$ the electron thermal velocity, where $k_{\mathrm{B}}$ is the Boltzmann constant, $T_{e}$ is the electron temperature and $m_{e}$ the electron mass. In presence of highly distorted EDFs, the absorption coefficient is decreased by a factor $f_{\mathrm{L}}$ (see Eq. 14) that reads:

$$
f_{\mathrm{L}}=\left[1-0.553 /\left(1+(0.27 / \alpha)^{0.75}\right)\right] .
$$

The $f_{\mathrm{L}}$ term depends on the fields from all beams and sheets. This is also the case of the CBET $\delta \epsilon_{i j}$ term described in the following section, which also depends on all sheets k-vectors. While one could use independent grids for each laser beam, computation of $f_{\mathrm{L}}$ requires that laser quantities are either shared on a common grid or interpolated for each beam. In standard hydrodynamic codes, a common grid is used, that also serves to compute ray trajectories, and that is also the hydrodynamic grid. This is not the case in the IFRIIT approach where we use a separate grid to trace rays (see Sec. 4.1) and a separate grid to compute fields (see Sec. 4.2).

\subsubsection{Cross beam energy transfer modeling}

CBET is a laser/plasma process by which overlapped laser waves may exchange energy, which occurs when beating waves create local density perturbations, acting as plasma gratings, that resonantly grow to large amplitudes when the corresponding matching conditions are met. Mathematically, each sheet may exchange energy with every other sheet, such that a single beam may exchange energy with itself. This is in general challenging to models in laser models implemented in RH codes because the concept of sheets is not compatible with approaches solely relying on the ray power equation (Eq. 9). In IFRIIT however, since we are constructing $\mathcal{M}$ from IRT, it is straightforward to separate these contributions, such that the total perturbation seen by sheet $j$ of beam $i$ is:

$$
\delta \epsilon_{i j}=\frac{\chi_{i, j} c^{2}}{4 \omega_{\mathrm{pe}}^{2}}\left[\sum_{\substack{l \in \text { beams } \\ m \in \text { sheets }}}\left|\mathbf{k}_{i j}-\mathbf{k}_{l m}\right|^{2}\left|u_{l m}\right|^{2} K_{l m}+\sum_{\substack{m \in \text { sheets } \\ m \neq j}}\left|\mathbf{k}_{i j}-\mathbf{k}_{i m}\right|^{2}\left|u_{i m}\right|^{2} K_{i m}\right],
$$

where $\mathbf{k}$ denotes the ray k-vector and we recall that $|u|$ is the field expressed in units of the oscillation velocity. $K$ designates a kinetic plasma response function that depends on the k-vectors of the respective ray fields, the hydrodynamic parameters, and the total laser intensity through the Langdon parameter $\alpha$ in case of non-Maxwellian distribution functions (see Refs. [9] and [18]). The $\chi_{i, j}$ coefficient in Eq. (17) represents the wave coupling decrease due to 
polarization effects. In IFRIIT, we implement both the unpolarized model [9] for beams smoothed with Polarization Smoothing, and a model which uses the wave polarization transported along rays and rotated through refraction. The latter will be detailed in a separate paper alongside results specific to polarization rotation effects.

We mention here that the CBET formulation and implementation in IFRIIT has been validated against the LPSE wave solver (see Ref. [19]), experimental data (see Ref. [18]), the Beamletcrosser code at LLE (D. Edgell, personnal communication), the VAMPIRE code at LLNL (see Ref. [20]) and the inline LASNEX model (D. Strozzi, personnal communication).

\subsubsection{Pump depletion iterations}

The perturbation terms to the permittivity introduced here, $f_{\mathrm{L}}$ and $\delta \epsilon_{i j}$, both depend on the values of fields. As such, the phase integration equation (Eq. (7)) must be solved iteratively with the equations for those perturbations (Eq. (16) and (17)). In the case of the Langdon effect on absorption and for the applications of interest presented here, $f_{\mathrm{L}}$ is close to 1 , such that the phase integration is updated only once. This is not the case for CBET, where significant depletion or amplification of the beams can occur. The iterations are then required for energy conservation. These iterations are referred to as pump depletion iteration, because they allow to account for the depletion of the pump energy in the CBET process.

The pump depletion iterations are controlled by assessing the convergence rate of exchanged energy through CBET. Denoting $\Delta P_{s, b}^{(\mathrm{i})}$ the absolute value of net energy exchanged by sheet $s$ of beam $b$ through CBET at iteration i, the following convergence criterion is defined:

$$
E_{\text {rel }}^{(i+1)}=\max _{\substack{s \in \text { sheets } \\ b \in \text { beams }}}\left|\Delta P_{s, b}^{(\mathrm{i})} / \Delta P_{s, b}^{(\mathrm{i}+1)}-1\right|<5 \times 10^{-4},
$$

with $P_{0, b}$ the initial power for beam $b$. Note that in this form, the criterion does not ensure energy conservation, only convergence of the iterative process.

In addition, it has been found that in situation of strong CBET, a damping term must be added in the update of the permittivity perturbation. The perturbation at step $i+1$ is updated as follows:

$$
\delta \epsilon_{i j}^{(\mathrm{i}+1)}=0.25 \delta \epsilon_{i j}+0.75 \delta \epsilon_{i j}^{(\mathrm{i})},
$$

where $\delta \epsilon_{i j}^{(i)}$ is the permittivity perturbation computed at previous step, and $\delta \epsilon_{i j}$ is the instantaneous perturbation computed as in Eq. (17).

\subsubsection{Frequency shift}

Until now we have retained the monochromatic assumption for the laser wave. While this is correct from a ray trajectory standpoint, small deviations in frequency can occur in plasmas, of the order of $0.1 \mathrm{~nm}$, which may affect CBET ( $K$ in Eq. (17) depends on the frequency $\omega$ on each contributing laser sheet). When an electromagnetic wave travels in a medium that exhibits time-varying electron density, the frequency of the wave can be up-shifted or downshifted. This effect originates from two contributions; first, the critical density where the light is reflected may be moving in time, and second, consecutive wave trains of light may see varying densities. Effectively, the frequency $\omega$ of the laser light obeys the following relation [21]:

$$
\frac{\mathrm{d} \omega^{2}}{\mathrm{~d} t}=\frac{\partial \omega_{p}^{2}}{\partial t}
$$

with $\omega_{p}=\sqrt{e^{2} n_{e} / m_{e} \epsilon_{v}}$ the electron plasma frequency and $\epsilon_{v}$ the vacuum permittivity. Making the change of variable $\mathrm{d} t=\mathrm{d} s / c \sqrt{\epsilon}=\mathrm{d} \tilde{\tau} / c$, and integrating for $\tilde{\tau}$ between 0 and $\tau$ one can obtain:

$$
\omega(\tau)^{2}=\omega(0)^{2}+\frac{e^{2}}{m_{e} \epsilon_{v} c} \int_{0}^{\tau} \frac{\partial n_{e}(\tilde{\tau})}{\partial t} \mathrm{~d} \tilde{\tau}
$$

Here, the value of $\partial n_{e} / \partial t$ can either be provided from the hydrodynamic calculation, or obtained from the continuity equation using $\nabla$. $\left(\mathbf{v} n_{e}\right)+\partial n_{e} / \partial t=0$ (obtained assuming that $\left.m_{e} \ll m_{i} / Z_{\mathrm{eff}}\right)$. The integral on the right-hand-side is evaluated in IFRIIT separately in each tetrahedron, against the ray trajectory. 


\section{Resolution of the ray equations in IFRIIT}

\subsection{Discussion of state-of-the-art resolution methods}

Over the years, many approaches to computing the laser/plasma coupling in RH codes have been developed. These can be broadly distributed in two categories for the equations resolved: ray power (Eq. 9) or ray amplitude (Eqs. 7 and 8). Stemming from these models are various methods to compute fields in space. A non-exhaustive list of ray-trace models implemented in the main ICF RH codes is as follows:

- Using the ray power approach:

- Rays are propagated forward into the RH mesh to obtain the power deposition binned in each cell (Eq. (9)). The ray field is then obtained using the electromagnetic energy conservation equation which relates absorbed power to laser intensity (see Sec. 5.1) [10]. Consequently, this field estimation method does not function in vacuum where there is no power absorbed, although the raytrace itself functions normally. This method also depends highly on the number of rays per cells. There is no treatment at caustics, where fields are non-divergent but erroneous.

- Rays are given a Gaussian intensity profile, which evolves following the paraxial complex geometrical optics equations $[22,23]$ as they propagate into the mesh. This method depends less on the number of rays, but has a stringent validity domain and is not valid at caustics either. It is also challenging to implement in 3-D due to the need to project the envelop of the rays onto the mesh.

- Ray intensities are computed along rays by dividing the ray power by the ratio of the cell volume to the path-length of the ray into the cells, thus obtaining a quantity dimensionally equivalent to an intensity [24].

- Ray intensities are computed along rays by dividing the ray power by a surface formed by adjacent rays $[25,26]$. This method diverges at caustics, where the maximum field is set with a limiter.

- Using the ray amplitude approach:

- Rays are propagated into the mesh carrying an amplitude and phase, and $D$ is approximated by tracking the position of adjacent rays with respect to a central ray [27]. While this approach allows to determine a ray field along the ray directly, it still depends on binning onto a mesh for CBET computations, where different rays must be able to exchange field values. Fields at caustics are divergent and limited with a parameter [16].

These approaches have in common the main following issues:

- fields at caustics are not determined consistently, with sometime the introduction of a free parameter used to tune the interaction,

- binning of the ray quantities on a static grid is required (at minimum for computing CBET and Langdon effect), which makes these methods depend strongly on the number of rays per cell,

- the binning grid is the hydrodynamic grid, such that the field computation is not dictated by laser-related parameters.

An exception to the last point is RH codes using adaptive mesh refinement, which sometimes allow grid refinements to be driven by laser field gradients. This in turn worsens the issue of number of rays per cell, thus requiring additional algorithms to converge the statistic.

The methods developed in this paper are intended to address these shortcomings. First, we use inverse ray-tracing (IRT) to directly compute the mapping $\left(\zeta_{1}, \zeta_{2}, \tau\right) \rightarrow(x, y, z)$. This has the follow advantages: (i) the ray amplitude (Eq. 8) can be precisely computed from the Jacobian, (ii) the ray field does not depend on a number of ray per cells, (iii) the incident and reflected fields are readily separated, (iv) an Etalon Integral method can be used to obtain the caustic field consistently, (v) the field can be reconstructed at higher order in space since there are no averaging volumes at play, and (vi) the field computation can easily be de-correlated from the hydrodynamic mesh. 


\subsection{Basic mesh structure for rays and subgrid model}

In IFRIIT, ray trajectories are computed on an unstructured 3-D tetrahedron grid designated as the raytracing mesh. Any gridding geometry may be used - e.g. cubic, cylindrical, spherical, arbitrary - as long as the mesh is constituted of tetrahedrons joined by their faces and without any degenerate (i.e. flat) cells. For the particular case of ICF calculations, we use a specific kind of spherical grid explicited in Sec. 4.1. The ray trajectory in the tetrahedrons is computed by making the approximation of constant permittivity gradient per cell. In that framework, the trajectory of a ray from position $\mathbf{r}\left(\tau_{0}\right)$ with momentum $\mathbf{p}\left(\tau_{0}\right)$ can be determined analytically:

$$
\begin{array}{r}
\mathbf{r}(\tau)=\mathbf{r}\left(\tau_{0}\right)+\mathbf{p}\left(\tau_{0}\right) \tau+\left(\tau^{2} / 4\right) \nabla \epsilon^{\prime}, \\
\mathbf{p}(\tau)=\mathbf{p}\left(\tau_{0}\right)+(\tau / 2) \nabla \epsilon^{\prime},
\end{array}
$$

where $\nabla \epsilon^{\prime}$ is a constant per cell 3-D permittivity gradient, determined from the values of $\epsilon^{\prime}$ at the 4 cell nodes. In that approximation, one can see from Eq. (21) that the ray trajectory is a parabola in each cell. In order to find the output position of the ray, one must find the value $\tau_{1}$ such that the ray intersects the first face found on its parabolic trajectory. This is done by changing the coordinate system locally and finding the intersections of the ray with all faces (inside and outside of the tetrahedron) and selecting the solution with the minimum positive traversal time (more details can be found in [19]). Once the face has been reached, the ray is traced onto the next tetrahedron following the same procedure. Note that in this approach, the permittivity along the ray trajectory is continuous across faces, such that there is no Snell-Descartes refraction at interfaces. Numerical tests have shown that such continuous permittivity profiles are required for the IRT procedure to converge.

Computation of the ray phase (Eq. (7)) and frequency (Eq. 20) for the computation of fields requires integration of given quantities along ray trajectories. These quantities are integrated assuming a linear dependency per cell of the coefficients being integrated (e.g. CBET gain), but not of the base hydrodynamic quantities themselves, as their combination would not be linear. This allows to use an efficient analytical formulation for the integration. The integral of a quantity $\chi$ along a ray trajectory in a cell reads:

$$
\int_{\tau_{0}}^{\tau_{1}} \chi(\mathbf{r}(\tilde{\tau})) \mathrm{d} \tilde{\tau}=\delta \tau\left[\chi\left(\tau_{0}\right)+\delta \tau \nabla \chi \cdot\left(\mathbf{p}\left(\tau_{0}\right) / 2+\nabla \epsilon^{\prime} \delta \tau / 12\right)\right],
$$

where $\delta \tau=\tau_{1}-\tau_{0}$ is the parametric traversal distance in the cell, $\nabla \epsilon^{\prime}$ is the associated constant permittivity gradient, $\nabla \chi$ is the constant gradient of quantity $\chi, \chi\left(\tau_{0}\right)$ is the value of $\chi$ at the ray entry point, and we have used the linear approximation $\chi(\mathbf{r})=\chi\left(\mathbf{r}_{1}\right)+\left(\mathbf{r}-\mathbf{r}_{1}\right) . \nabla \chi$ with $\mathbf{r}_{1}$ the location of node number 1 of the cell. In practice, $\chi$ may here be $\epsilon^{\prime \prime}=\epsilon_{0}^{\prime \prime} f_{\mathrm{L}}+\delta \epsilon^{\prime \prime}$ (for integration of Eq. (7)), or $\partial n_{e} / \partial t$ (for integration of Eq. (20)).

\subsection{Inverse ray-tracing and observed quantities}

In the IRT approach, the goal is to build the multi-sheet mapping $\mathcal{M}$ evoked in Sec. 2.2, i.e. associating each observation point with the phase space parameters that allow to trace rays from the initial ray surface up to it. Here we designate as $\mathbf{R}\left(\zeta_{1}, \zeta_{2}, \tau\right)$ the ray-tracing function that computes the final position of a ray traced from $\left(\zeta_{1}, \zeta_{2}, 0\right)$ up to $\left(\zeta_{1}, \zeta_{2}, \tau\right)$. At the end point, we obtain the laser quantities; values of the field $u$ (Eqs. 7 and 8), frequency $\omega$ (Eq. 20) and k-vector (Eq. 6). The principle is then to observe these ray parameters at specific points. By arranging the observation points in a specific gridding pattern, one can then set up interpolators to reconstruct continuously the ray parameters across the plasma. This contrasts with what is usually done in RH codes which rely on forward ray-tracing (FRT), where the ray parameters must be binned onto a mesh in order to reach converged values. The binning process itself produces discontinuous (constant-per-cell) quantities which heavily depend on the number of rays per cells, as illustrated in Sec. 5.2.

The procedure to construct $\mathcal{M}$ is as follows. For each observation point $\mathbf{r}_{0}$ contained in anservation grid where the laser quantities are sought, we find the ray parameters $\left(\zeta_{1}, \zeta_{2}, \tau\right)$ such that the trajectory of a ray starting at the initial surface ends up at $\mathbf{R}\left(\zeta_{1}, \zeta_{2}, \tau\right)=\mathbf{r}_{0}$. This is achieved through a multi-dimensional Newton solver that minimizes $\left\|\mathbf{R}\left(\zeta_{1}, \zeta_{2}, \tau\right)-\mathbf{r}_{0}\right\|_{\infty}$. The procedure is in three steps. First, the positions of the laser caustics in phase space are recorded in order to separate the contributions of each sheet to the total field. This is done by looking for parameters where $D(\tau)$ changes sign, due to a phase shift of $-\pi / 2$ induced by the reflection at the caustic [28]. 
Second, a guess map is constructed to initialize the Newton step. This is done by sampling each sheet over a range of phase space parameter and constructing a 3-D Delaunay triangulation of the resulting set of points. Last, the Newton iterations are conducted. Solutions are sought to a precision such that $\left\|\mathbf{R}\left(\zeta_{1}, \zeta_{2}, \tau\right)-\mathbf{r}_{0}\right\|_{\infty}<\varepsilon_{\mathrm{IRT}}$, where $\varepsilon_{\mathrm{IRT}}=\lambda_{0} / 5$ outside caustic regions and $\lambda_{0} / 50$ inside caustic regions, with $\lambda_{0}$ the vacuum wavelength of the laser (typically 351 $\mathrm{nm})$. A sample algorithm of the IRT step is given in Appendix A.

\subsection{Model limitations}

\subsubsection{Limits on the description of laser sheets}

The current numerical implementation of IRT described in the paper is illustrated for two laser sheets; the incident and reflected fields. The IFRIIT code, however, was written in a more general manner with the number of sheets being a parameter. For a laser/plasma configuration with $n$ laser sheets, the code is in theory able to handle each of the separate $n$ sheets and $n-1$ associated caustics. In practice, numerical experiments have shown that in situations where refraction rates are high, such as in the cases presented in Sec. 5.2, ray trajectories in the second sheet are noisy. This noise tends to build up after reflection on caustics. Tracking a third sheet in such cases would be highly challenging due to the corresponding higher ray trajectory noise. This noise transcribes in the Jacobian computations and usually prevents the IRT from converging, as well as introducing oscillations in the amplitude term (Eq. (8)). In addition, the Jacobian noise will prevent the Etalon Integral method from yielding physical values (see Sec. 2.3). This is because the ray parameters in Eq. (10) must be known to sufficient accuracy to avoid the divergence.

As such, describing more than two laser sheets can be done in the current framework only for permittivity gradients slowly deviating from the linear case. One can, for example, imagine a waveguide profile approached by two linear slopes. A laser beam incident at an angle in the waveguide would produce multiple sheets and caustics. Going beyond this limitation would require to increase the order of the subgrid model for the permittivity, which may require to change the base elements of the raytracing mesh (tetrahedrons). Notably, while the permittivity profile is continuous across tetrahedron faces, the derivative is not continuous. Recovering continuity of the derivative across interfaces would greatly reduce raytracing noise.

\subsubsection{Limits on the modeling of caustics}

In this paper we have considered a model for caustics fields based on fold-type caustics. The Etalon Integral we have used for this is the so-called Uniform Airy Asymptotics, which involves two rays. In practice, various integrals exist for the various kinds of caustics. One could in theory implement models for other caustic types, which would require to track more rays per observation points in that case. As an example, we can mention cusp-type caustics which involve 3 rays, and swallowtail, wave crest, and hair-type caustics which involve 4 rays [29, 14].

\subsubsection{Summarized limits on the use of the IRT model in IFRIIT}

Due to the limits exposed on the accuracy of tracking more than 2 sheets and on the modeling of caustics in the current IFRIIT framework, the IRT field solver cannot be applied in a variety of cases. A non-exhaustive list of such cases relevant to direct-drive studies is as follows:

- Simulations with large 3-D feature that introduce complex caustics or sheet configurations, such as target holders. In that case, the use of FRT may be preferred. While CBET can still be computed in that case with FRT (see Sec. 5), it likely will not be to the same accuracy and with the same performances.

- Simulations with concave permittivity profiles such as those created from small wavelength perturbations on targets. In that case, it is preferred to start calculations using the FRT field solver, and switch to IRT once the coronal plasma, which usually features convex permittivity profiles, is well developed.

- Simulations with surface defects, which cannot be meshed with sufficient precision to ensure IRT convergence. Similarly to the previous point, the beginning of the interaction can be modeled with FRT until the coronal plasma is developed. 


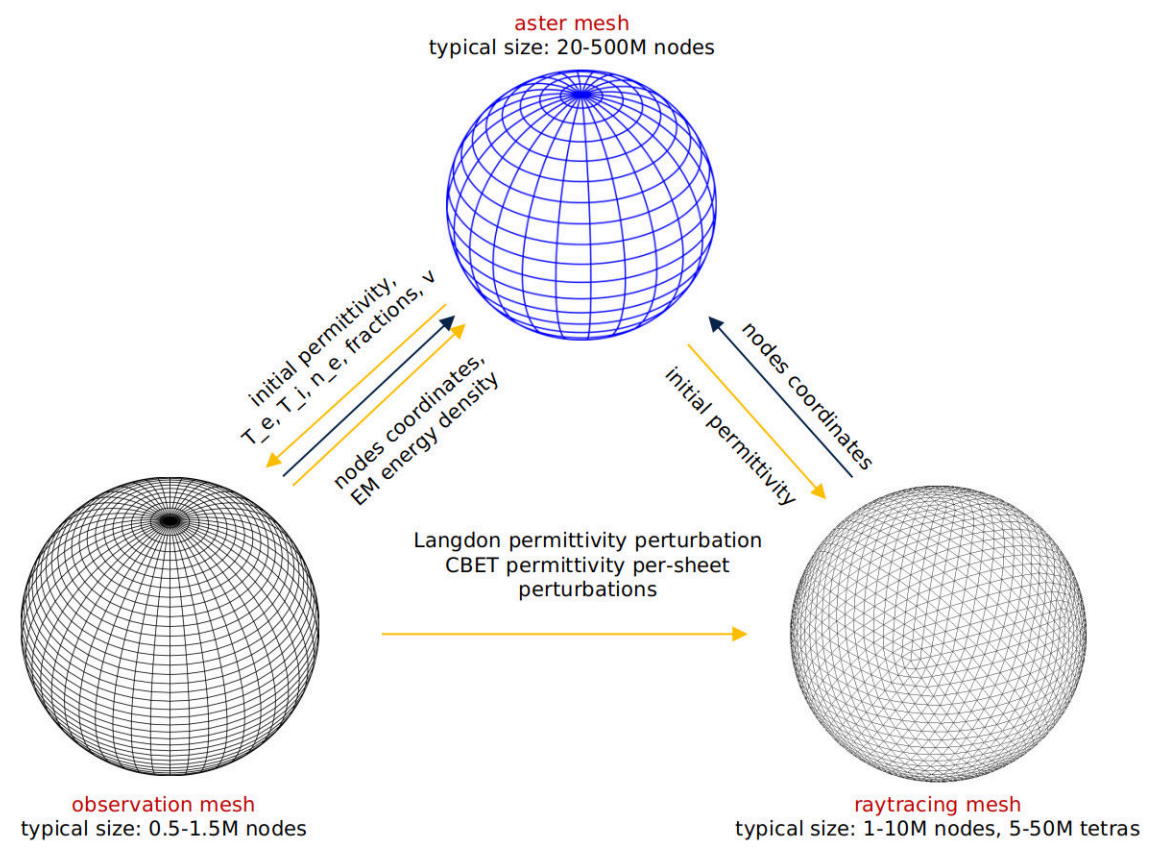

Figure 3: General principle of the grid coupling between ASTER (blue grid) and IFRIIT (black grids). The ASTER mesh is a high resolution polar grid used to solve the equations of radiative hydrodynamics. The IFRIIT raytracing mesh is a spherical geodesic grid used to trace rays. The IFRIIT observation mesh is a polar mesh where fields are computed. The permittivity $\epsilon$, plasma temperatures $T_{e}$ and $T_{i}$, electron density $n_{e}$, plasma velocity $\mathbf{v}$ and material fractions $f$ are defined on the observation mesh (and interpolated from the ASTER mesh). These are used for computation of the Langdon effect, CBET coupling coefficients and the fields. The plasma permittivity is defined onto the raytracing mesh (also interpolated from the ASTER mesh), so that the trajectory equations (Eqs. 6) can be resolved. The permittivity perturbations from Langdon effect and CBET, computed on the observation mesh, are interpolated onto the raytracing mesh for integration of the phase equation (Eq. 7). Finally, the EM energy density, obtained at the nodes of the observation mesh, is back-interpolated at the cell vertices of the ASTER mesh. The result is then tri-linearly integrated from the vertices onto the cell volume. Information exchange between the three grids in the ASTER+IFRIIT operation, indicated with arrows. Only the exchanges to and from the ASTER mesh are MPI communications. Note that since the latter is block-decomposed, communications with IFRIIT occur between specific ranks only (see parallelization scheme in Sec. 4.3). Yellow arrows indicate that an interpolation is performed (see interpolation methods in Appendix B).

\section{Coupling of ASTER and IFRIIT}

The ASTER and IFRIIT code coupling operates by exchanging information between various grids where the respective equations of the two codes are resolved. This is detailed in Fig. 3, alongside the communication and data storage of the grids. ASTER employs a high resolution polar mesh where the equations of radiative hydrodynamics are resolved. IFRIIT employs two meshes, a geodesic tetrahedron mesh where rays are traced (see Sec. 4.1), and a polar mesh where ray fields are evaluated and non-linear couplings computed (see Sec. 4.2). The use of different grids allows to use different parallelization methods for each code, so that their concurrent use relies on a heterogeneous parallelization scheme (see Sec. 4.3). We also use load balance to ensure optimum operation between the two codes, as shown in Sec. 4.4. A specific warmup procedure is implemented to minimize noise in the early laser-plasma interaction (Sec. 4.5). Finally, the code operation diagram is summarized in Sec. 4.6.

\subsection{Icosahedral ray-tracing mesh}

\subsubsection{General icosahedral mesh}

As mentioned in Sec. 3.2, rays are traced in a tetrahedral tessellation of the coronal plasma. For optimum numerical accuracy, the tetrahedron nodes of the tessellation should be aligned with iso-contours of the plasma permittivity profile. In case of ICF implosion experiments, targets are shells or solid balls, and hence spherically symmetric. In addition, they are driven as uniformly as possible, so that the plasma profile gets as close as possible to 1-d radial profiles. As such, it is natural to seek an arrangement of the tetrahedron vertices in spherical layers of nodes at discrete radial coordinates. 
The simplest of such spherical grids is the so-called polar mesh, a tessellation of the sphere using octahedric elements obtained by sampling the three base axis of the spherical coordinate system $(r, \theta, \phi)$, where $\theta$ refers to the polar angle and $\phi$ is the azimuthal angle. Here, $\theta$ is taken to be zero along the $z$-axis of the corresponding $(x, y, z)$ Cartesian coordinate system and $\phi$ is taken to be zero along the $x$-axis. The main drawback of these grids is the presence of degenerate volume elements: hexahedric cells sharing four vertices at $\theta=0$ or $\theta=\pi$ with $r \neq 0$, pentahedric cells sharing four vertices at $r=0$ and tetrahedric cells combining vertices at $\theta=0$ or $\theta=\pi$ with $r=0$. Such meshes cannot be decomposed purely in tetrahedric cells without introducing flat tetrahedrons to resolve connectivity issues on cell boundaries. In addition, volume elements in polar meshes have a significantly non-uniform distribution, with narrow cells around poles and wide cells at the equator, which could potentially introduce a spherical harmonics mode 2 in the laser deposition distribution. This is avoided by using a geodesic grid for the raytracing step, as described below. Conversely, the mesh where the value of the field is computed is taken to be a polar mesh (see Sec. 4.2), for efficiency and simplicity of back-and-forth interpolations with the ASTER mesh, which is also polar.

The IFRIIT raytracing mesh nodes are arranged in spherical layers, each following a geodesic icosahedron. Contrary to geodesic meshes used e.g. in meteorology, the number of vertices in each spherical layer increases with increasing radius. This allows to fill the space between layers with a non-degenerate tetrahedral-octahedral tessellation. The mesh is constructed as follows. The starting point is an icosahedron, i.e. an assembly of 20 master tetrahedrons sharing a central vertex (see Fig. 4(a)). We define three vectors in each master tetrahedron along which nodes are sampled: $\mathbf{e}_{r}$ is an outward radial vector, $\mathbf{e}_{t 1}$ and $\mathbf{e}_{t 2}$ are transverse vectors that follow great circles of the sphere. Each of the master tetrahedron is sampled with $N_{r}$ radial layers. Vertices in radial layer $i$ (where $i \in\left[1, N_{r}\right]$ ) are a regular triangulation obtained from edge subdivision of the generating triangle into $N_{t}(i)=i$ sub-edges, such that radial layer $i$ possesses $(i+1)(i+2) / 2$ vertices per master tetrahedron. Following this method of construction, we have $N_{t 1}\left(N_{r}\right)=N_{t 2}\left(N_{r}\right)=N_{i}$ sub-edges on the outer boundary of the mesh per master tetrahedron. This is illustrated in Fig. 4 (b).

The space between adjacent layers is filled with tetrahedrons using the available vertices. This is achieved by using a tetrahedral-octahedral honeycomb with a ratio of 2 tetrahedrons for 1 octahedron. An illustration of this process is given in Fig. 4 (c). Each octahedron in the honeycomb is split into 4 sub-tetrahedrons by choosing an arbitrary diagonal. Since the chosen diagonal is systematic across the master tetrahedron, this procedure does introduce a preferential direction in the mesh. Finally, tetrahedrons in the layer are connected to each others through their shared faces, and further connected to upper and lower layers. The process is repeated for the 20 master tetrahedrons, which are then assembled in the icosahedron geometry. Faces at the outer interfaces of each master tetrahedron are connected and redundant vertices are removed from the grid. Finally, vertices positions on each radial layer are projected onto the corresponding sub-sphere radius. An example low resolution mesh is given in Fig. 5. In this meshing approach, the total number of vertices scales as $\propto\left(10 N_{r}\left(5+N_{r}^{2}\right)\right) / 3$ while the total number of tetrahedrons is simply $20\left(N_{r}-1\right)^{3}$ (assuming $N_{r}>1$ ). Finally, since the number of vertices increases per spherical layer, the angular resolution increases in the outward radial direction as $\simeq\left(5\left(N_{t}(i)+1\right)\right) / 360$ cells/degree. This is contrary to polar meshes which have constant angular resolution as a function of radius.

\subsubsection{Specific icosahedral mesh for spherical calculations}

In the configuration of spherical laser target interaction, the laser only propagates in the coronal plasma, between the vacuum $(\epsilon=1)$ and solid $(\epsilon=0)$ densities. In addition, the characteristic length of permittivity variations, or scalelength, is highly inhomogeneous across the corona; short next to the solid density and significantly longer closer to the vacuum. In order to optimize the IFRIIT grid for these specific spherical calculations, the icosahedron raytrace mesh is optimized with (i) a hollow core and (ii) radially varying distribution of the spherical layers.

Hollow core. The interior of the grid is truncated, such that spherical layers now have a transverse number of faces per master tetrahedron of $N_{t 1}(i)=N_{t 2}(i)=N_{t}^{(\mathrm{s})}+(i-1)$, with $N_{t}^{(\mathrm{s})}$ a value specified at the solid density and $i \in\left[1, N_{r}\right]$. This value drives the coarsest angular resolution of the grid. Typical calculations employ $N_{2 \pi}^{(\mathrm{s})}=360$ cells per great circles at the solid density, with $N_{t}^{(\mathrm{s})} \sim N_{2 \pi}^{(\mathrm{s})} / 5=72$. The radial extent of the grid is determined by radial coordinates $R_{\text {vac }}$ and $R_{\text {sol }}$ such that $\min _{\theta, \phi}(\epsilon(r))>0.99$ for $r>R_{\text {vac }}$ and $\max _{\theta, \phi}(\epsilon(r))<0$ for $r<R_{\text {sol }}$, where the minimum and maximum values are taken with respect to the angular coordinates. 
(a)

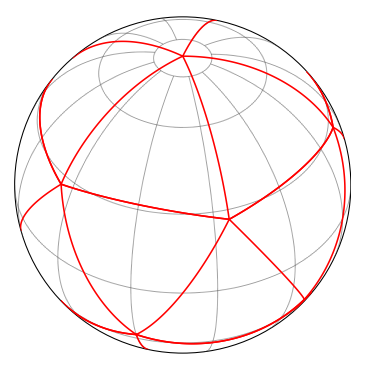

(b)

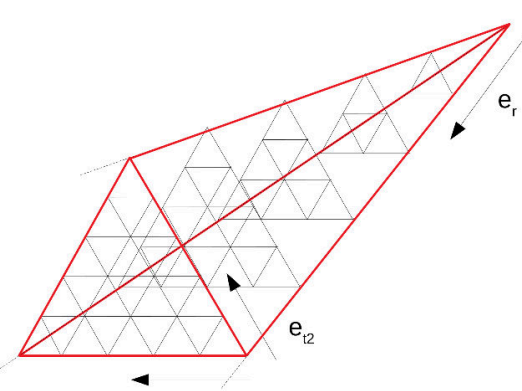

$e_{\mathrm{t} 1}$ (c)

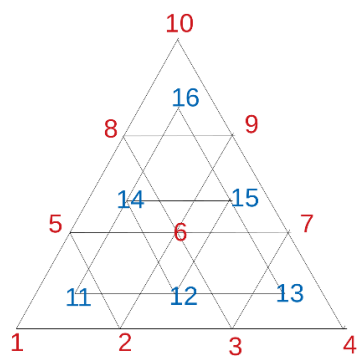

Figure 4: (a) Icosahedron tiling of a sphere. The external facets of the master tetrahedrons are shown in red. (b) Subdivision of a master tetrahedron in layers of nodes, each following from the subdivision of a triangle to increasing order. (c) Representation of two overlapped layers of nodes. Assuming the center node of the icosahedron forms layer 0, the layers shown are here layers 2 and 3, in blue and red, respectively. Tetrahedrons used to fill these layers are as follows. "Upward" tetrahedrons: $[1,2,5,11],[2,3,6,12],[3,4,7,13],[5,6,8,14],[6,7,9,15]$ and $[8,9,10,16]$, , downard" tetrahedron: $[6,12,15,14]$, octahedrons: $[2,5,6,11,12,14],[3,6,7,12,13,15]$ and $[6,8,9,14,15,16]$. The octahedrons are then split into tetrahedrons along the diagonals: $[2,14],[3,15]$ and $[6,16]$.

(a)

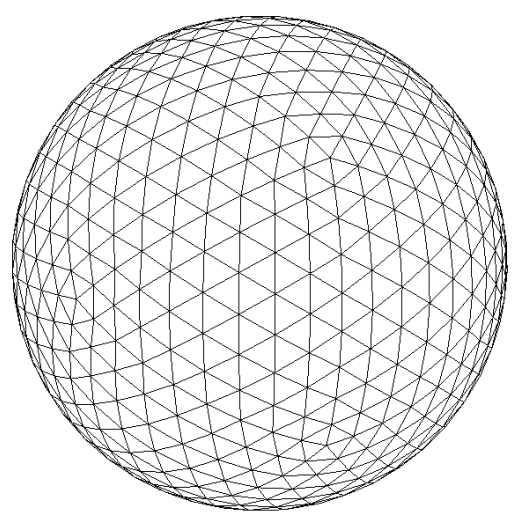

(b)

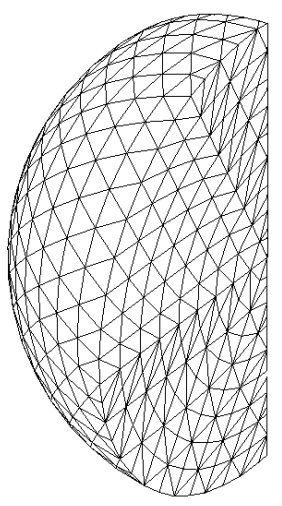

Figure 5: (a) Geodesic raytracing mesh generated with approximatively 50 nodes per great circle on the outer boundary, and 10 radial layers (visible in (b)). (b) Cut of the raytracing mesh showing the tetrahedron tessellation. Orientation shifts in the various master tetrahedrons are visible as changes in meshing patterns. 
(a)

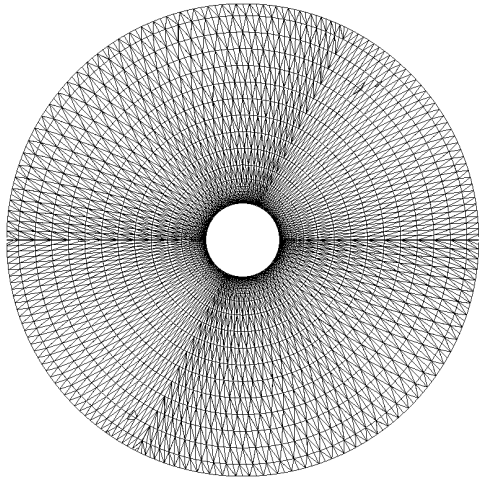

(b)

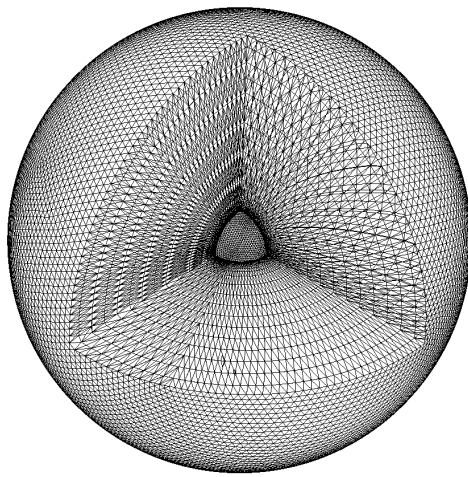

(c)

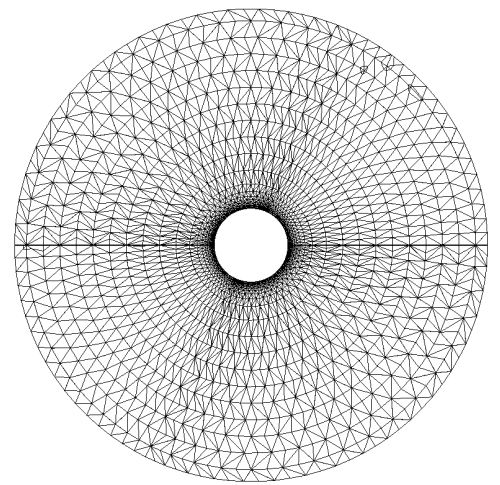

Figure 6: Equatorial cut (a) and exploded view (b) of a raytracing mesh obtained in the spherical configuration using the hollow core and variable radial meshing approach. (c) Equatorial cut of a raytracing mesh using the aforementioned methods and the alternated layers approach in addition. Both meshes were obtained with $N_{2 \pi}^{(\mathrm{s})}=90, N_{s}=10, L_{\min }=\lambda / 10$ and $L_{\max }=500 \lambda$. The underlying permittivity profile is that given in the test cases of Sec. 5.2.

Variable radial distribution. The coronal plasma is radially sampled with a varying step in order to increase accuracy in regions of short permittivity scalelength and decrease memory and computing costs in regions of long permittivity scalelength. The radial sampling follows a meshing function $L(r)$ which dictates the spherical layer spacing:

$$
\left\{\begin{array}{l}
g(r)=\max _{\theta, \phi}\left(\|\nabla \epsilon(r, \theta, \phi)\|_{2}\right), \\
L_{\mathrm{g}}(r)=\left|1 /\left(g(r)+\varepsilon_{\mathrm{L}}\right)\right| \\
L(r)=\min \left(L_{\max }, \max \left(L_{\min }, L_{\mathrm{g}}(r) / N_{s}\right)\right),
\end{array}\right.
$$

where $g(r)$ is the permittivity gradient maximum amplitude computed from the RH code, $L_{\mathrm{g}}(r)$ is the permittivity scalelength associated to the gradient profile $g(r), N_{s}$ is a refinement parameter and $\varepsilon_{\mathrm{L}}=10^{-10}$ is a safety parameter. $L_{\min }$ and $L_{\max }$ are minimum and maximum radial spacings, typically set to $\lambda / 10$ and $500 \lambda$, respectively. This meshing function, used with $N_{s} \geq 10$, ensures that permittivity gradients are correctly meshed and that the linear-pertetrahedron subgrid model remains pertinent. An illustration of a raytracing mesh obtained in this manner is shown in Fig. $6(a, b)$.

Alternated layers. The truncated geodesic grid has an increasing angular resolution with each radial layer. Such increase in resolution in the low density part of the corona is not required, and can lead to large raytracing meshes for long plasma coronas. An alternative meshing configuration is to retain the hollow core and variable radial meshing, but to alternate layers such that the number of nodes per shell increases from shell $i$ to $i+1$ and then decreases from $i+1$ to $i+2$, and so on. Effectively, the tetrahedrons in the layer separated by shells $i+1$ and $i+2$ are radially inverted with respect to the layer separated by $i$ and $i+1$. Consequently, there is a constant number of tetrahedrons per layer. This configuration is illustrated in Fig. 6 (c). In this meshing approach, both the number of vertices and of tetrahedrons scale as $\propto N_{r}$. Similarly to the polar mesh, the angular resolution is now constant with radius. Because this approach was only recently implemented, results presented throughout the paper mainly use the first approach, without alternated layers. Numerical tests using the alternated layers approach are evoked in: the single-beam test case (Tab. 1) and the CBET/no-CBET inline test case (see timings in Fig. 9 and the discussion on mesh modes in Sec. 5.4.2).

\subsection{Observation mesh}

\subsubsection{Purpose of the observation mesh}

The icosahedron mesh presented in the previous section is optimized for raytracing: it ensures that the 3-D permittivity profile is correctly discretized such that the ray trajectory equations (Eq. 6) can be resolved to sufficient 
accuracy. In particular, the computation of the Jacobian for IRT (Sec. 3.3) and for the amplitude term (Eq. 8) is sensitive to noise in the raytracing. As such, sufficient raytracing mesh resolution must be employed; a typical raytracing mesh in an inline calculation will possess in the range of [1M-10M] nodes and [5M-50M] tetrahedrons.

Conversely, knowledge of laser-related quantities (field, k-vector, frequency) is not required at such high resolutions because the typical scalelength of variation of laser fields are large compared to the raytracing mesh resolution. As an example, in order to resolve a spherical harmonics mode 30 and requiring 5 cells for the smallest mode, of the order of 150 cells per great circle are required, compared to the minimum of 360 used for the raytracing mesh. A similar argument can be made for the resolution in the radial direction.

As such, it is advantageous to compute laser-related quantities on a mesh that is different from the raytracing mesh. Since we are using IRT, we can effectively compute the field quantities at any location within the raytracing mesh. This mesh where fields are computed is denoted observation mesh. The computation of the Langdon effect coefficient (see Sec. 2.4.1) and the CBET exchange coefficient (see Sec. 2.4.2) is also conducted onto the observation mesh. Note that no equations are actually integrated over the observation mesh. It is an intermediary data structure representing fields in space and used to compute costly multi-beam processes.

\subsubsection{Structure of the observation mesh}

Since the observation mesh does not require to be triangulated in 3-D, it is possible to use a simple polar $(r, \theta, \phi)$ grid. However, interpolation is still required to project laser quantities back onto the raytracing mesh for integration of Eq. (7). The polar grid has an advantage in that framework, namely that efficient interpolation methods can be used by leveraging the rectilinear property of the $\theta$ and $\phi$ axes. The observation mesh is defined by the number of cells used in the angular directions, $N_{\theta}^{\text {obs }}$ and $N_{\phi}^{\text {obs }}$, and a radial spacing function for the layers. For the latter, we use the same formulation as in Eq. (24), with its own set of parameters $L_{\mathrm{max}}^{\mathrm{obs}}, L_{\mathrm{min}}^{\mathrm{obs}}$ and $N_{s}^{\mathrm{obs}}$. Laser parameters computed on the observation mesh are projected onto the raytracing mesh when integration of the phase equation is required. Interpolation methods used to communicate between the three grids are described in Appendix B

\subsubsection{IFRIIT to ASTER source term}

While hydrodynamics quantities are sent from ASTER to IFRIIT, IFRIIT communicates back a source term for the collisional absorption. In steady state, the conservation equation for the laser energy is $\nabla . S=-\omega_{0} \epsilon^{\prime \prime} W$, with $W=\epsilon_{0}|E|^{2} / 2$ the EM energy density and $\mathcal{S}=\epsilon_{0} \mathbf{k} c \sqrt{\epsilon^{\prime}}|E|^{2} /(2|\mathbf{k}|)$ the Poynting vector. Note here that we have not included beam exchange terms from CBET or Langdon effect, since those are essentially accounted for in the pump depletion iterations when computing the fields. The heat source term $\Omega_{i}^{\mathrm{hs}}$ from the laser absorption in ASTER cell $i$ reads:

$$
\Omega_{i}^{\mathrm{hs}}=\sum_{b \in \text { beams }} \frac{m_{e}^{2} \omega_{b, 0}^{3}}{2 e^{2} \mu_{0}} \epsilon_{b, i}^{\prime \prime} \int_{V_{i}} \sum_{s \in \text { sheets }}\left|u_{s}^{2}\right| \mathrm{d} V,
$$

where $\mu_{0}$ is the vacuum permeability, $\epsilon_{b, i}^{\prime \prime}$ is the imaginary part of the permittivity seen by beam $b$ and taken at the center of ASTER cell $i, \int_{V_{i}}$ denotes an integral over the cell volume, and we are assuming $\omega \simeq \omega_{0}$ for the purpose of energy deposition. Because the ASTER and IFRIIT grids do not coincide in the radial direction, using $\epsilon^{\prime \prime}$ at the IFRIIT nodes would lead to energy deposition in the first vacuum cell which may cause issues with equations of states. This is prevented by using the ASTER cell-centered value for $\epsilon^{\prime \prime}$. The volume integration method used for Eq. 24 is detailed in Appendix C.

\subsection{Parallelization method}

ASTER, as many RH codes, is MPI-parallelized using domain decomposition, in a pure-MPI parallelization approach. Each MPI rank holds a part of the hydrodynamic mesh, or block, which slightly overlaps with its neighbor through ghost cells. Most MPI communications happen in the ghost cells in order to exchange boundary information for the hydrodynamic evolution. Domain decomposition is well adapted to RH because the load is well balanced between the cells. This is not the case for laser raytrace in general, since a laser beam may propagate in only a few blocks if it is narrow. In that framework, rays are propagated in a given block, reach a boundary, and are then transmitted to the next block. Even if the target is uniformly irradiated, such that rays propagate in many blocks at once, 
Parallel run: hydrodynamics + IFRIIT threads
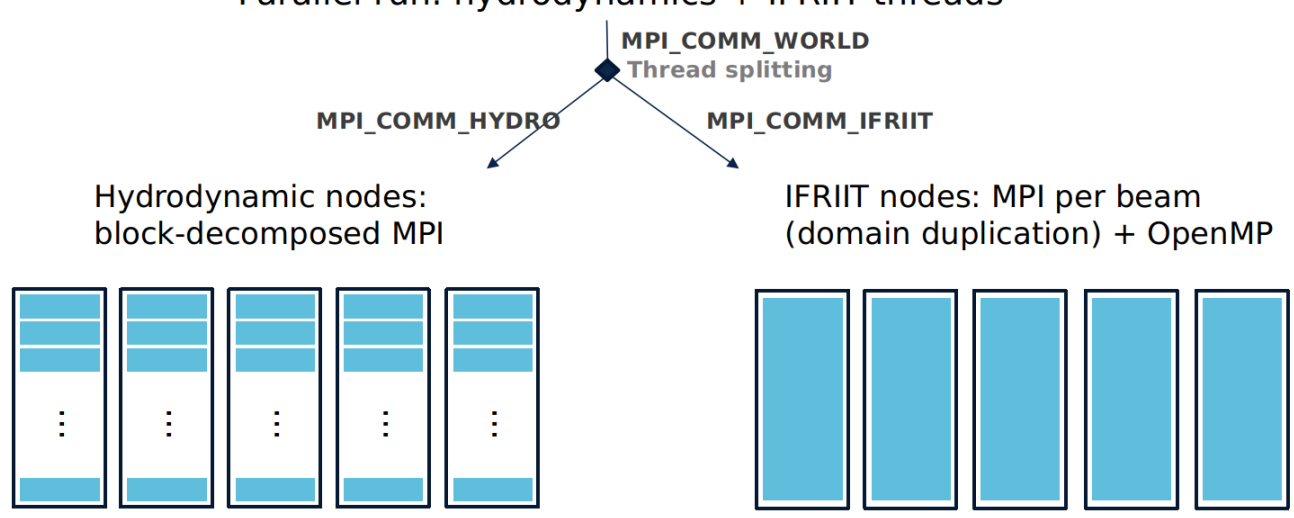

Figure 7: Illustration of the parallelization scheme employed in ASTER+IFRIIT. Each rank is represented as a blue bar, and black rectangles represents computing nodes. It is not compulsory to fill the last hydrodynamic node. In addition, several laser MPI ranks can be used on the same node. Finally, hydrodynamic and laser ranks can be mixed, although the decomposition illustrated here is the most efficient.

this parallelization scheme remains sub-optimal since blocks deeper in the mesh will still be waiting for rays at their boundaries.

Since the IFRIIT mesh is independent from the hydrodynamic mesh, we use a different parallelization method that is more balanced, namely, hybrid MPI-OpenMP parallelization. IFRIIT uses a domain-duplication strategy where each MPI rank holds the entire meshes in memory (raytracing and observation meshes). This is made possible because these meshes are optimized to be memory efficient; the radial gridding is adaptive and only the coronal plasma is meshed. Note that this would not be possible using the ASTER mesh, which has tens of times larger node numbers than the IFRIIT raytracing mesh (see Fig. 3). In this approach, each MPI rank is assigned a single beam: a 60 beams calculation then requires 60 MPI ranks. On a given IFRIIT MPI rank, we make use of OpenMP shared memory parallelism to further speed up operations. This implies that at maximum parallelism, each IFRIIT rank is placed on a single compute node and uses OpenMP parallelism in that node.

The specificity of the parallelization scheme in the ASTER+IFRIIT code is that it simultaneously uses two types of parallelism. This approach is called heterogeneous parallelism. The rank repartition of an heterogeneous ASTER+IFRIIT calculation is illustrated in Fig. 7. Typically, cluster nodes are filled by pure-MPI ASTER ranks sequentially until the desired number is met. Then, the IFRIIT MPI ranks are assigned to the remaining nodes such that sufficient OpenMP threads can be used in each. While ASTER calculations can require between 100 to 10000 MPI ranks depending on the mesh resolution employed, higher resolutions being used for study of high modes hydrodynamic instabilities, the IFRIIT calculation always uses approximately the same number of threads. This is because the IFRIIT mesh adapts to the hydrodynamics, and the coronal plasma has approximatively the same scalelength whatever the resolution is used in the hydrodynamics. In practice, IFRIIT is designed to be used with 20 to 40 threads per MPI rank. An OpenMP scaling of IFRIIT is shown on Fig. 8.

\subsection{Load Balance and concurrent operation}

In conventional RH codes, laser deposition is computed consecutive to the other physics modules. While this is the most accurate method, it is often unnecessary. The typical timescale for evolution of hydrodynamics processes in long-pulse laser / plasma interactions is of the order of a few picoseconds (it is dictated by the laser pulse variations). However, typical CFL conditions imply that RH codes timestep at a fraction of a picosecond. In the ASTER case, and for a typical implosion, the timestep for a low resolution case $(128 \times 256$ angular cells, $0.2 \mu$ m minimum radial resolution) is in the $0.02-0.2 \mathrm{ps}$ range. While small steps are required to capture correctly shock dynamics and hydrodynamic instabilities, the coronal plasma itself is rather steady state during the main pulse, when the laser energy does not change much. Consequently, the IFRIIT step is not conducted in sequence to the hydrodynamics, but asynchronously. This gives more computational time to conduct a single IFRIIT step, and is made possible by the separation of the IFRIIT and RH computational threads. 


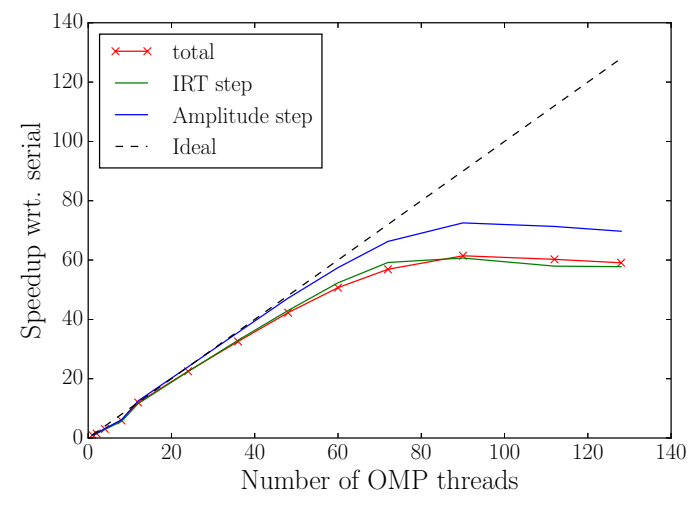

Figure 8: OpenMP strong scaling for an offline IFRIIT spherical test case. The problem size is kept constant and the number of OpenMP ranks increased. In that particular case, the IRT step drives the scaling.

The following conditions are used to determine when ASTER must trigger an IFRIIT calculation:

- if $t+\mathrm{d} t>\mathrm{d} t_{\max }$, with $\mathrm{d} t_{\max }$ set to the order of $1-2 \mathrm{ps}$,

- if the laser power at $t+\mathrm{d} t$ is $2 \%$ larger or lower than at $t$,

- if the lasers were off at $t$ and are no longer off at $t+\mathrm{d} t$, or

- if the code is in warmup mode, where high accuracy is required.

In all other cases, including when lasers are off, IFRIIT is bypassed, and the source term applied in the hydrodynamics is that of the previous IFRIIT step scaled to the current laser power. This strategy is necessary given the significantly higher computational cost of an IFRIIT step compared to an ASTER step. An example of load-balancing is given in Fig. 9, where the time spent for a single IFRIIT steps is compared to that of several ASTER steps, in between two updates. At ideal load-balance, the two metrics should be similar, meaning that the two codes do not idle waiting for each other. In practice, ASTER, in the current set-up, is faster at early times and slower later on, due to the CFL requiring smaller hydrodynamic steps at that time.

In this framework where the codes run concurrently, and given that the IFRIIT grid is de-correlated from the hydrodynamic grid, the cost of IFRIIT operation is roughly the same whatever the chosen hydrodynamic resolution is. This is a great advantage for this approach: in a standard RH code, if one were to increase the resolution by a factor of 2 in each angular direction, the number of rays should be increased by a factor of 4 to match the same ray statistics per cell. In addition, increasing resolution often requires increasing the number of MPI threads, often worsening the speedup provided by the parallelism. This is not the case here: the hydrodynamic code resolution can be increased freely and the IFRIIT cost (in terms of number of MPI ranks and total compute time) will remain the same.

\subsection{Warmup}

During the first instants of the laser/plasma interaction, hypotheses made for the derivations of the geometrical optics equation break down. Notably, the slowly varying envelope approximation, which requires that the permittivity scalelength be much larger than the wavelength, breaks down. In addition, conducting IRT steps in the highly narrow spherical layer of the first few picoseconds is challenging, and precision issues limit the convergence of the algorithm on the sides of the target where rays are grazing. This is mainly because of the aspect ratio of the tetrahedron cells, which are significantly shorter in the radial direction during warmup compared to the angular direction. As en example, consider a radial layer of $50 \mathrm{~nm}$ (which depends on the hydrodynamics mesh resolution) and a spherical target of 500 microns radius. The circumference of the sphere is then of the order of $31.410^{6} \mathrm{~nm}$, that is 63000 times the radial resolution. Memory constraints will severaly limit the ability to produce a good quality mesh at these instants, and IRT convergence will be challenging. When the plasma heats up, the radial extent of the layer increases 
(a)

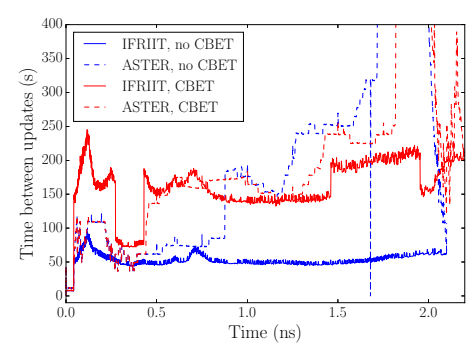

(b)

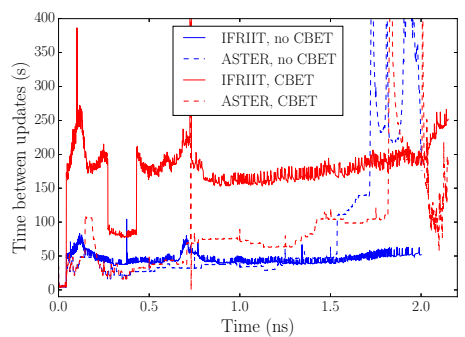

(c)

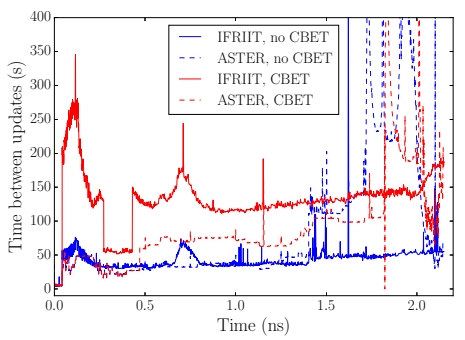

Runtime (hr)

\begin{tabular}{|c|c|c|c|c|c|c|c|}
\hline & & & & \multicolumn{4}{|c|}{ Runtime (hr) } \\
\hline & & & & Hollow + & ble radial & + alternate & layers \\
\hline CPU kind & $\begin{array}{c}\text { hydro } \\
\text { MPI ranks \# }\end{array}$ & $\begin{array}{c}\text { laser } \\
\text { MPI ranks \# }\end{array}$ & $\begin{array}{l}\text { laser OpenMP } \\
\text { threads/rank \# }\end{array}$ & no CBET & CBET & no CBET & CBET \\
\hline $\begin{array}{c}\text { 2x24-cores } \\
\text { Intel Skylake@2.7GHz } \\
\text { (AVX512) }\end{array}$ & 96 & 60 & 24 & 78.2 & 87.9 & & \\
\hline $\begin{array}{c}\text { 2x64-cores } \\
\text { AMD Rome@ } 2.6 \mathrm{Ghz} \\
\text { (AVX2) }\end{array}$ & 256 & 60 & 42 & 48.3 & 80.5 & 41 & 63.3 \\
\hline
\end{tabular}

Figure 9: Synchronization time:, i.e. time spent by ASTER (dashed lines) between two IFRIIT calls, and by IFRIIT (solid lines) between two ASTER calls. These timings include the time spent by each code waiting for the other code, and all communication times. The test cases correspond to the configuration presented in Sec. 5.3, with (red) and without (blue) CBET. The hollow core with variable radial distribution raytracing mesh was used for IFRIIT in (a,b), while the alternated meshing was used in (c). In all cases, the ASTER mesh is $128 \times 256$ cells in the angular directions and 707 cells in the radial direction, decomposed on (a) 96 MPI ranks and (b,c) 256 MPI ranks. The IFRIIT calculation involves 60 ranks (beams) with (a) 24, and (b,c) 42 OpenMP threads per beam. Calculations were performed on (a) 2x24-cores Intel Skylake@2.7GHz (AVX512) CPU nodes and (b,c) 2x64 AMD Rome@2.6 Ghz (AVX2) CPU nodes. Total runtimes are reported in the table.

and the memory constraints are alleviated as the mesh quality improves. Finally the preferential direction introduced in the icosahedral mesh (see Sec. 4.1.2) induces asymmetries in the collisional absorption (in the integration of Eq. (7)) which makes the scattered field non-symmetric. While this asymmetry is not visible when the mesh possess enough layers, it is significant at startup. As such, IFRIIT cannot be used in its normal operation mode during the plasma creation at startup. Note here that this is also a time where various physics model break down: the plastic shell (irradiated by the laser) is actually transparent at early times, cold EOS and opacities are not properly modeled, ionization models are not valid, and so on.

In order to avoid imprinting the target at early time with numerical artifacts, a specific warmup procedure is used as long as the radial extent of the coronal plasma (from $\epsilon_{v}=0.99$ to solid at $\epsilon_{s}=0$ ) is less than $20 \lambda_{0}$. The procedure, illustrated in Fig. 10, possesses two phases: in the first phase, the first solid-facing cell in ASTER has a permittivity above $\epsilon_{v}$. During this time, the fields are computed as follows: (i) the scattered field is deactivated but the total absorption value is conserved, thus avoiding the numerical scattering asymmetry, (ii) the IFRIIT mesh has two radial layers with refraction deactivated and the layers are shifted to center the absorption rate onto the vacuum-facing solid ASTER cell and (iii) non-linear processes are deactivated (CBET, Langdon effect, $\mathrm{d} n_{e} / \mathrm{d} t$ shift). During the first phase of warmup, the beam profiles are essentially projected directly onto a solid sphere which radius is that of the critical density. This phase typically lasts less than 2 ps.

The warmup procedure moves to the second phase when the first solid-facing cell has filled up sufficiently (below $\epsilon_{v}$ ). During the second phase (see Fig. 10[top right]), the IFRIIT mesh is constituted of three layers; the first is inside the solid density, the second is centered at $\epsilon=0.1$ and the third at $\epsilon=\epsilon_{v}$. For the field computation step, the fields are directly projected onto the second layer, ignoring refraction and the scattered field once again. This radial arrangement ensures that in the back-projection to ASTER, a source term will be projected from solid to vacuum, scaled by the proper permittivity. The second phase typically lasts $30-40 \mathrm{ps}$, point at which the normal IFRIIT operation starts. Finally, during the entire warmup procedure, a spherical harmonics $\left(Y_{l m}\right)$ smoothing filter is applied layer by layer to the source term computed on the ASTER mesh, filtering modes above $l=30$. This filter artifacts that can occur when detecting the lit/shadow boundary at early times. 

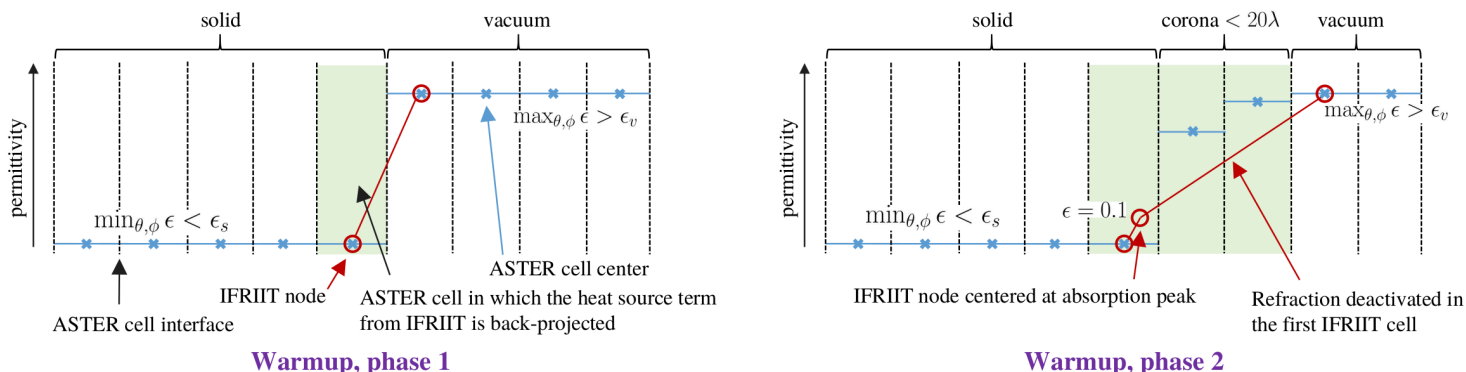

Warmup, phase 1

Warmup, phase 2

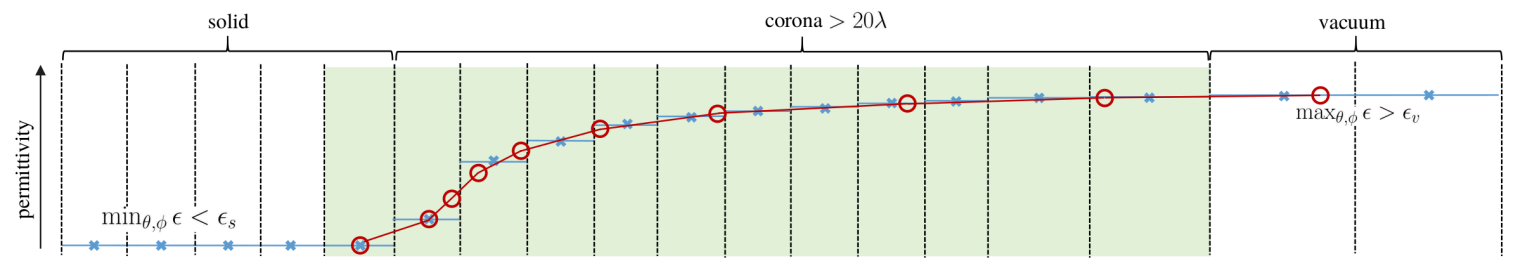

Normal operation

Figure 10: Schematic representation of the radial meshing and settings used in IFRIIT during the warmup phase compared to normal operation. Blue lines and crosses represent the ASTER cell-centered permittivity values. Red circles represent the IFRIIT nodes, and red lines represent the linear permittivity values in the cells. The green colored region indicate the ASTER cells where the IFRIIT back-projection will lead to non-zero heat source. In the normal operation mode, the meshing functions (for raytracing and observation) follow the distributions given in Sec. 4.1.2

\subsection{Operation diagram}

When operating ASTER and IFRIIT simultaneously, the codes are compiled as a single executable. The main MPI communicator is then split in: (i) ranks executing both the radiative hydrodynamics operations (ASTER code) and some of the IFRIIT input preparation, and (ii) ranks executing the laser computations (IFRIIT code). A schematic summary of the main operations is given in Fig. 11. The amount of MPI communications between ASTER and IFRIIT is kept low by only sending the relevant data to the relevant ranks, and by operating the data transfers in single precision (except for node positions and the plasma permittivity). Aside from initialization, the communication between the codes starts by IFRIIT sending back the spatial laser absorption density, computed from the plasma parameters taken at time $t_{1}$. This density map is interpolated on the ASTER grid at $t_{2}$ with $t_{2}$ being chosen according to parameters given in Sec. 4.4. The $t_{2}$ plasma parameters are then sent to IFRIIT, interpolated on the new mesh created from the updated permittivity profile. While IFRIIT computes the new laser step, ASTER performs several hydrodynamic steps up to $t_{3}$, using a saved heat source map that is scaled to the laser power at each step. A breakdown of the computing cost of the main steps in IFRIIT is shown in Fig. 12 and illustrates that the majority of the time is spent in computing fields (IRT and amplitude steps) and pump depletion for CBET, and not in communicating with ASTER or interpolating results and inputs.

\section{Numerical experiments}

We present two sets of applications of the IFRIIT and ASTER+IFRIIT code. The first one is an offline (i.e. IFRIIT only - without hydrodynamic evolution) test case of 60 beams interacting in a spherically symmetric profile. In this case, we assess the performances of IRT, and compare it to the FRT method. The second set of application is inline modeling of cryogenic implosions using ASTER+IFRIIT.

\subsection{Formulation for FRT-based field estimation}

It is useful here to compare the benefits and drawbacks of using IRT compared to a conventional FRT approach which uses grid-binning to define laser quantities. In general, in FRT-based models, rays are propagated from the initial ray surface into the mesh and laser parameters are binned onto the mesh cells. Several methods to compute 


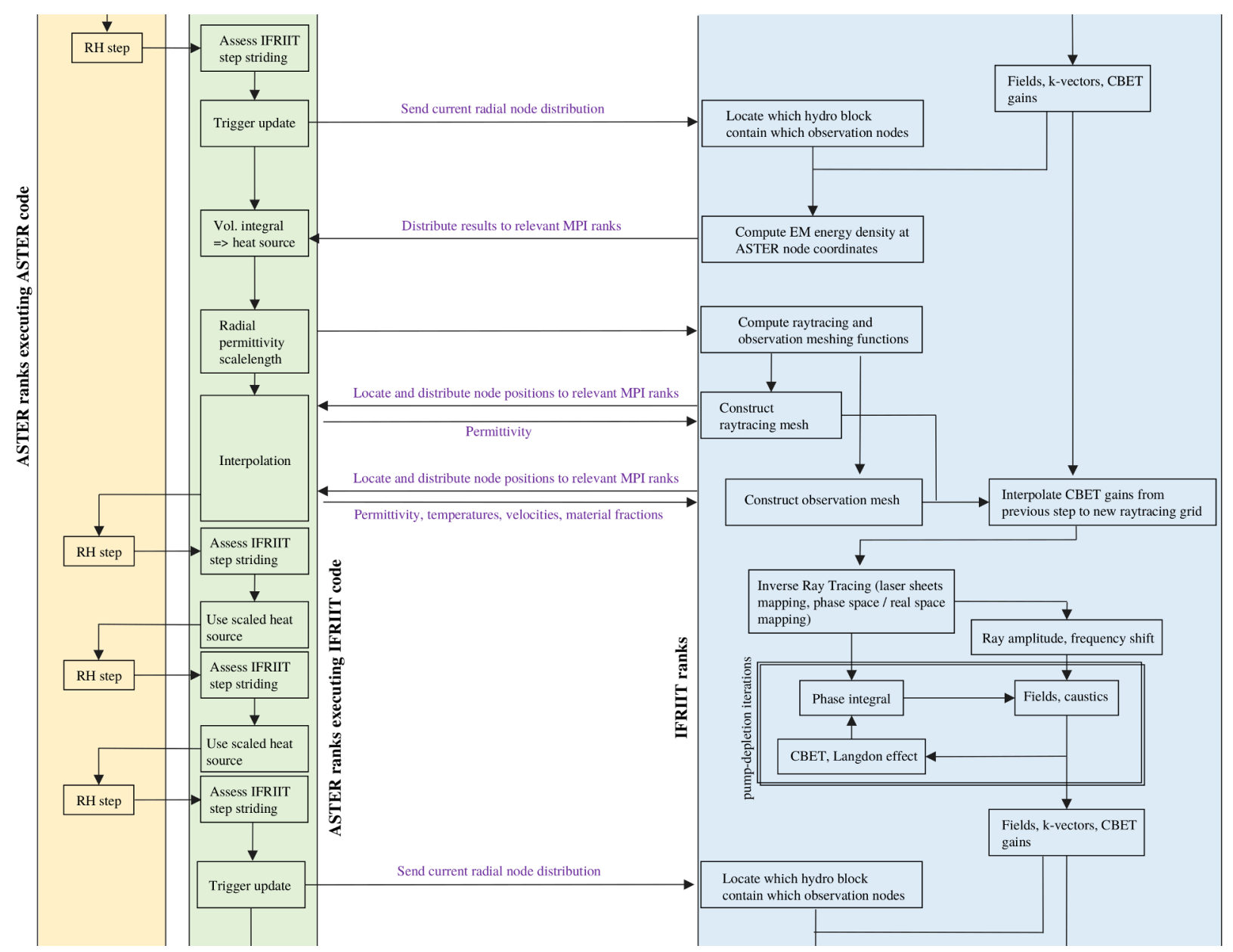

Figure 11: Schematic representation of the operation principle of the ASTER+IFRIIT coupling. Operations in the orange and green columns are carried out by ASTER MPI ranks, with orange being ASTER source code and green being IFRIIT source code. Note that ranks executing the orange and green operations are the same, and not separate ranks. Operations in the blue columns are carried out by IFRIIT MPI ranks and is IFRIIT source code. MPI communications between the codes are represented with arrows between the ASTER (green) and IFRIIT (blue) ranks. These also corresponds to communications shown in Fig. 3.

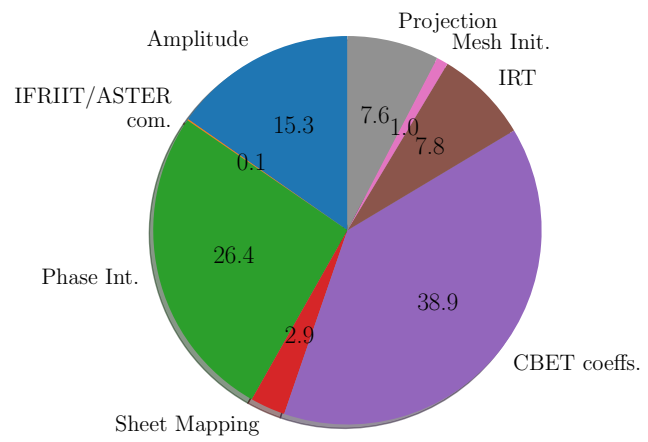

Figure 12: Breakdown of the time spent in the main sections of the IFRIIT algorithms, in $\%$ of the total runtime. Results are reported for the configuration presented in Sec. 5.3 for a case with CBET. The ASTER/IFRIIT communications represent $0.1 \%$ of the runtime. 
fields using this binning process exist for FRT, as was presented in Sec. 3.1. Here, we implement the method based on computing laser intensity from collisional absorption. In each tetrahedron $i$ of the icosahedron mesh, an estimate of the cell-centered field $\left|\hat{u}_{i}\right|$ and $\mathrm{k}$-vector $\hat{\mathbf{k}}_{i}$ is computed is as:

$$
\begin{aligned}
\Delta P_{i} & =\sum_{j \in \text { rays }} P_{i j, 0} \exp \left[-k_{0} \int \epsilon^{\prime \prime}\left(\mathbf{r}_{\mathbf{i j}}(\tilde{\tau})\right) \mathrm{d} \tilde{\tau}\right], \\
\left|\hat{u}_{i}\right| & =\frac{e}{m_{e} c \omega_{0}^{3 / 2}} \sqrt{\frac{2 \Delta P_{i}}{\Delta V_{i} f_{L} \epsilon_{0, i}^{\prime \prime} \epsilon_{0}}}, \\
\hat{\mathbf{k}}_{i} & =\frac{\left\langle\mathbf{p}_{\mathbf{j}}\left(\tau_{i j} / 2\right)\right\rangle \sqrt{\epsilon_{i}^{\prime}}}{\left|\left\langle\mathbf{p}_{\mathbf{j}}\left(\tau_{i j} / 2\right)\right\rangle\right|}
\end{aligned}
$$

where $\Delta P_{i}$ is the total power deposited in cell $i, P_{i j, 0}$ denotes the input power of ray $j$ in cell $i$, obtained from integration of Eq. $9, \mathbf{r}_{\mathbf{i j}}(\hat{\tau})$ is the associated trajectory in the cell, $\Delta V_{i}$ is the cell volume, $\left\langle\mathbf{p}_{\mathbf{j}}\left(\tau_{i j} / 2\right)\right\rangle$ denotes the averaged ray momentum over rays in cell $i$ and taken at the midpoint of each ray trajectory, and we have used the steady state conservation equation (see Sec. 4.2.3) to derive (26). It must be noted here that $\epsilon_{0, i}^{\prime \prime} f_{L}$ in Eq. (26) does not include the contribution from CBET explicitly since it is factored in $\Delta P_{i}$ directly.

Since quantities in IFRIIT require to be node-centered, notably for computation of the integral (Eqs. (9) and (25)) and the CBET and Langdon coefficients (see Secs. 2.4.2 and 2.4.1), $\left|u_{i}\right|$ and $\mathbf{k}_{i}$ are recast to nodes by using a simple smoothing operator:

$$
F_{n}=\frac{\sum_{i \in \mathcal{N}_{n}} \hat{F}_{i} / d_{i, n}^{2}}{\sum_{i \in \mathcal{N}_{n}} 1 / d_{i, n}^{2}},
$$

where $F_{n}$ denotes a node-centered value of $F$ at node $n, \hat{F}_{i}$ is a cell centered value of $F$ at cell $i, d_{i, n}$ is the distance between node $n$ and cell center $i$, and $\mathcal{N}_{n}$ is a set of neighbor tetrahedrons that share node number $n$.

The accuracy of the reconstructed maps for $|u|$ and $\mathbf{k}$ depends on: (i) the size of the tetrahedrons, with smaller elements allowing to resolve finer structures, and (ii) the number of rays per cell, which allows better statistics for the binning in Eqs. (25) and (27). Here, we cannot make use of a decorrelated observation mesh. As such, laser quantities are computed directly on the raytracing grid. The number of rays used is controlled by a parameter $\beta=N_{\text {rays }} / N_{\text {ext.faces. }}$. which represents the approximate ratio of the number of rays (per beam) to the number of external mesh faces facing the beam profile. $\beta$ is an approximation of the number of rays per cells in absence of refraction, and as such $\beta \gg 1$ is desired. Here we underline, for clarity, that rays are traced into the same raytracing mesh be it either for FRT and IRT.

\subsection{Offline comparison of IRT vs FRT}

The fields reconstructed by FRT and IRT are compared in an offline test-case relevant to the OMEGA [13] laser geometry and target scales. The plasma is a spherically symmetric profile extracted from a 1-D LILAC simulation of a typical OMEGA ICF experiment. The corresponding hydrodynamic parameters are given in Fig. 13. Two cases are considered; first, interaction of one beam in the plasma, including self-CBET of the incident and reflected field, and second, a 60 beams interaction. In both cases, the damping term in $\epsilon_{0}^{\prime \prime}$ is computed using the standard inverse Bremsstrahlung rates [30] and the Langdon effect on absorption is included. The spot profiles considered are so-called SG5 phaseplates, which generate super-Gaussian laser spots of order 5.2 and 1/e radius of $358 \mu \mathrm{m}$.

\subsubsection{Single-beam interaction at high-intensity}

Here we consider a single SG5 OMEGA beam interacting in the spherical profile. The beam intensity is set to $3 \times 10^{15} \mathrm{~W} / \mathrm{cm}^{2}(10 \mathrm{TW})$ to enhance non-linear effects. Note that this is past the maximum power for a single OMEGA beam. Simulation results are compared between the FRT and IRT methods. In the FRT case, the approximate number of rays per cell $\beta$ is varied from 50 to 250 , while for IRT, only the radial resolution of the observation mesh is varied. The coupling is computed with Langdon effect on absorption and with or without CBET. Results are reported in Tab. 1 and Fig. 14. 


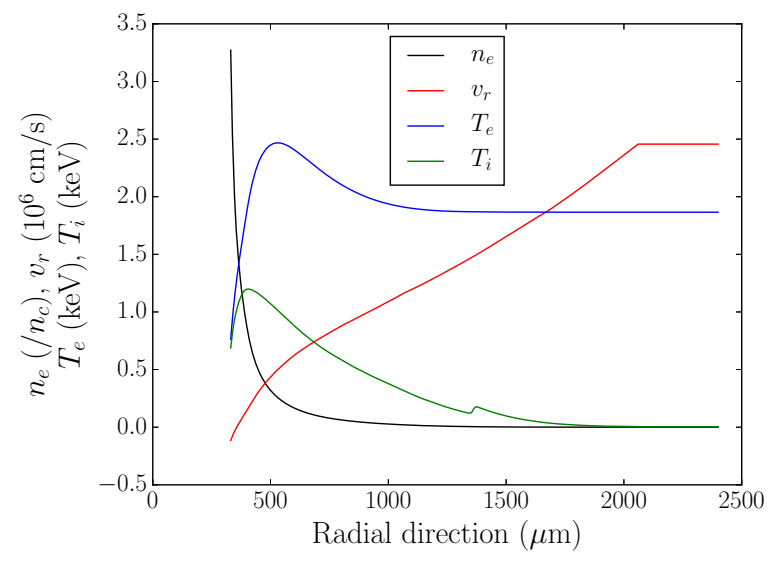

Figure 13: Hydrodynamics parameters considered in the offline test case, as a function of the target radius in micron. The parameters are: the electron number density (black), radial velocity (red), electron (blue) and ion (green) temperature. The material is $\mathrm{C}_{57.45} \mathrm{H}_{42.55}$ with $\langle Z\rangle=3.87$ up to $1360 \mu \mathrm{m}$, followed by fully ionized aluminum.

\begin{tabular}{|c|c|c|c|c|c|c|c|c|}
\hline Geodesic raytracing mesh type: & \multicolumn{6}{|c|}{ Hollow core + variable radial meshing } & \multicolumn{2}{|c|}{+ alternated layers } \\
\hline $\begin{array}{c}\text { Field solver } \\
\text { Solver parameters }\end{array}$ & $\begin{array}{c}\text { FRT } \\
\beta=50\end{array}$ & $\begin{array}{c}\text { FRT } \\
\beta=150\end{array}$ & $\begin{array}{c}\text { FRT } \\
\beta=250\end{array}$ & $\begin{array}{c}\text { FRT } \\
\beta=400\end{array}$ & $\begin{array}{c}\text { IRT } \\
N_{s}^{\mathrm{obs}}=25\end{array}$ & $\begin{array}{c}\text { IRT } \\
N_{s}^{\mathrm{obs}}=50\end{array}$ & $\begin{array}{c}\text { IRT } \\
N_{s}^{\mathrm{obs}}=25\end{array}$ & $\begin{array}{c}\text { IRT } \\
N_{s}^{\mathrm{obs}}=50\end{array}$ \\
\hline runtime $(\mathrm{s})$ [CBET+Langdon] & 2641 & 3465 & 6640 & 7240 & 141 & 267 & 96 & 138 \\
\hline absorption fraction (\%) [CBET+Langdon] & 53.52 & 67.80 & 70.41 & 71.40 & 71.84 & 71.78 & 71.77 & 71.57 \\
\hline energy conservation error (\%) & 31.27 & 9.77 & 2.01 & 1.58 & 0.51 & 0.18 & 0.17 & 0.18 \\
\hline$N_{\text {rays }}(\mathrm{M})$ & 7.81 & 23.44 & 39.06 & 62.5 & 1 & - & - & - \\
\hline$N_{\text {nodes }}^{\text {obs }}(\mathrm{M})$ & - & - & - & - & 0.47 & 0.86 & 0.47 & 0.86 \\
\hline runtime $(\mathrm{s})$ [Langdon] & 90 & 190 & 285 & 426 & 56 & 82 & 46 & 62 \\
\hline absorption fraction (\%) [Langdon] & 91.52 & 91.76 & 91.76 & 91.76 & 92.07 & 92.03 & 92.04 & 91.99 \\
\hline
\end{tabular}

Table 1: Runtime performances for the single-beam high intensity self-interaction case. Results are given for FRT and IRT solvers. In all cases, the raytracing mesh uses the radial meshing function given in Eq. 24 with $N_{s}=50, L_{\min }=0.05 \lambda, L_{\max }=500 \lambda$, and $N_{2 \pi}^{(s)}=360$. Using the standard hollow geodesic grid configuration, the mesh consists of 31.9M tetras and 5.58M nodes. In the alternated layer configuration (see Sec. 4.1.2), the mesh consists of $16.9 \mathrm{M}$ tetras $2.86 \mathrm{M}$ nodes. Note that there is no notion of number of rays for the IRT, and similarly, there is no notion of number of observation nodes for the FRT. For the IRT setup, the angular meshing of the observation mesh employs $N_{\theta}=90$ and $N_{\phi}=180$. Simulations are conducted on 2x64 AMD Rome@2.6 Ghz (AVX2) CPU nodes with 24 OpenMP threads per beam. 


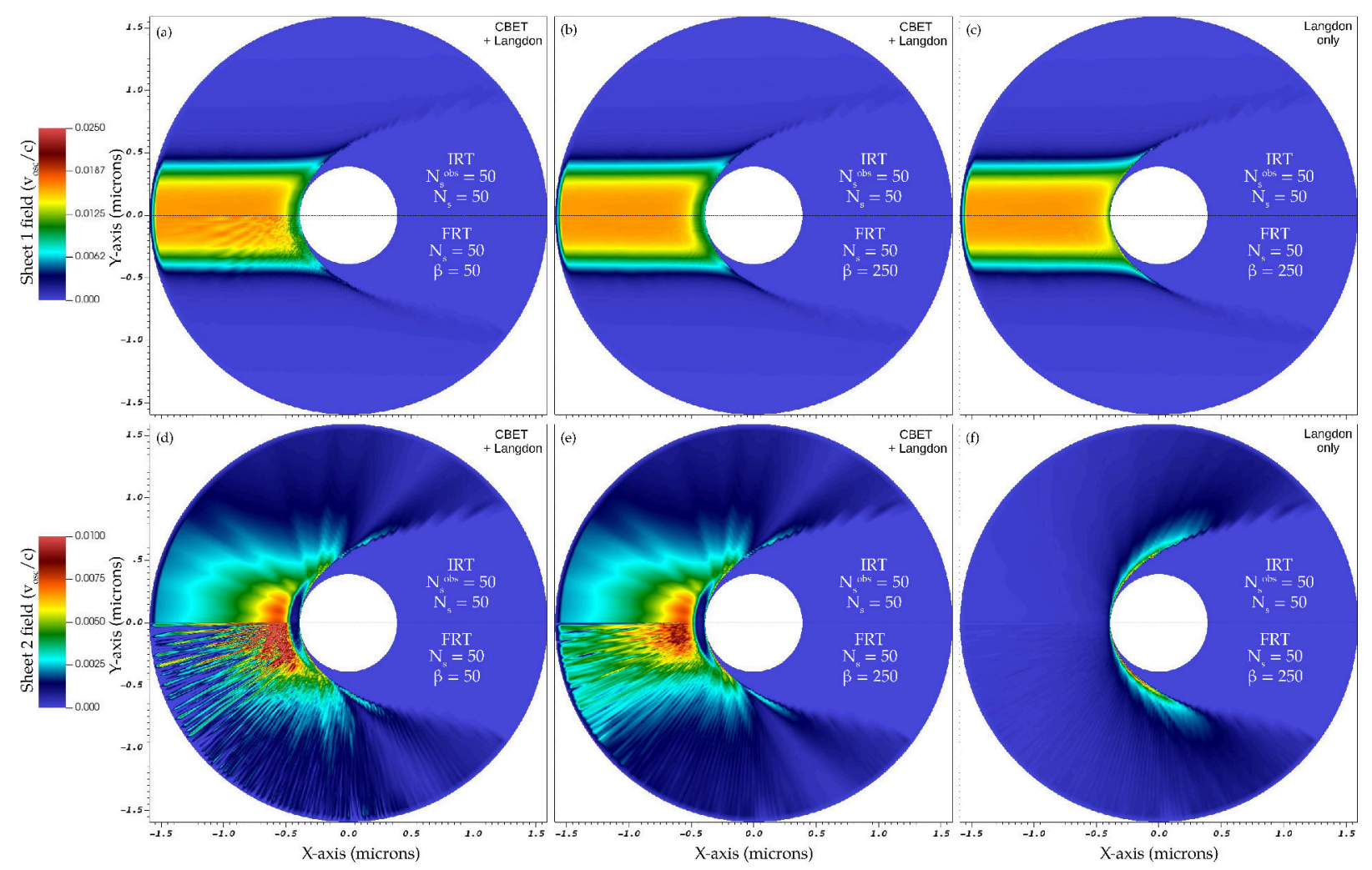

Figure 14: Slices of the 3-D fields for $(a, b, c)$ the incident field, or sheet 1, and (d,e,f) the reflected field, or sheet 2 . The top and bottom half in each plot show results using IRT and FRT, respectively. Cases $(\mathrm{a}, \mathrm{b}, \mathrm{d}, \mathrm{e})$ are with CBET and Langdon effect on absorption, and without CBET in (c,f).

The FRT and IRT methods have similar performances in the case without CBET and with Langdon effect on absorption. This is expected because the Langdon effect depends weakly on the laser field, such that the accuracy of its calculation is not as critical as for CBET. Conversely, when adding the CBET effect, significant differences in performances are observed between FRT and IRT. The former converges slowly towards the IRT solution, and requires runtimes in the order of 50 times that of the IRT case. As is illustrated in Fig. 14, this is a consequence of the noise in the scattered field. The latter is caused by two effects; first, the high refraction rates which diminish the ray statistics in cells for FRT, and second, modulations created by the mesh structure which tend to bunch trajectories of reflected rays. The latter is also present in IRT although in a lesser extent, as can be seen in Fig. 14 (d) and (c) [top-half].

The shorter runtime of IRT is due to two factors: first, the low noise of IRT induces faster convergence of the pump depletion algorithm, and second, by separating the computation of the amplitude and phase terms, the IRT saves significant time. This is also for two reasons: first, because the amplitude term does not depend on the fields it does not need to be recomputed at each iteration, and second, because the update of the field phase only requires one to two rays per node of the observation mesh. On the contrary, the computation in the FRT case requires to re-propagate all rays at each iteration of the pump depletion algorithm. Another consequence of the lower order field reconstruction with FRT can be seen in the CBET energy conservation error, which is significantly higher with FRT than with IRT (see Tab. 1). For that particular test case, about 250 rays per cells are required for convergence of the FRT, which represents here 40M rays per beam. On the contrary, the IRT calculation requires much less resources and converges rapidly.

The significant performance difference between FRT and IRT in those test cases illustrate some of the severe short-comings of conventional FRT methods for CBET in 3D, notably: the high dependancy on ray statistics (which is rapidly critical in direct drive due to the high refraction rates, especially in 3D), the need to retrace all rays to iterate pump depletion. Our example shows that a typical implementation of FRT for CBET in 3D direct-drive applications may not be sufficient for accurate calculations in highly non-linear regimes. However, that does not preclude to adapt 
(a)

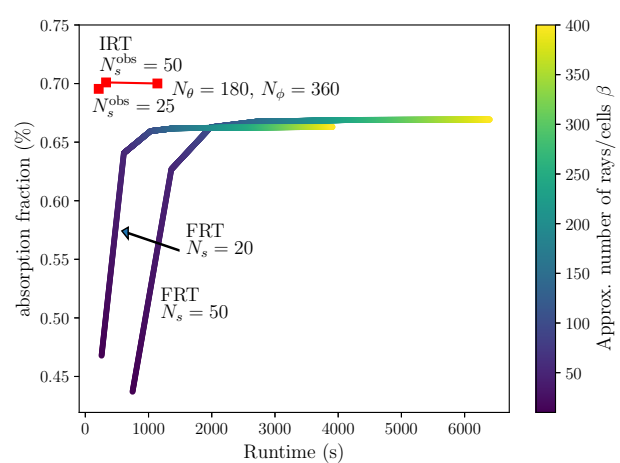

(b)

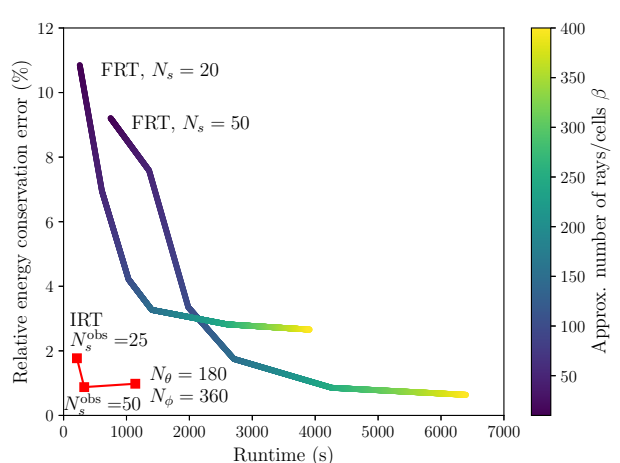

Figure 15: (a) Integrated absorption fraction normalized to the incident laser energy, and (b) relative energy conservation error, as a function of total runtime. Unless specified otherwise in the figures (notably for values of $N_{s}, N_{\theta}$ and $N_{\phi}$ ), the raytracing mesh and observation mesh are setup in the same way as reported in Tab. 1. Results from the IRT are shown in red and results from the FRT are shown from blue to yellow depending on the value of $\beta$. Simulations are conducted on 2x64 AMD Rome@ 2.6 Ghz (AVX2) CPU nodes with 24 OpenMP threads per beam.

future FRT models to remedy to those issues and recover good convergence properties.

\subsubsection{0-beams interaction}

We now consider a 60 beams case in the OMEGA chamber layout [13] with the hydrodynamic parameters kept the same. The intensity per beam is set to $1.05 \times 10^{14} \mathrm{~W} / \mathrm{cm}^{2}(0.36 \mathrm{TW}$ per beam), a standard value for implosions on OMEGA during the main drive. Simulations are compared for FRT and IRT, with CBET and Langdon effect on absorption enabled. A summary of the results for the integrated absorption and the CBET energy conservation error are presented in Fig. 15. Contrary to the single beam case, the FRT and IRT do not converge toward the same integrated absorption value, with a $3 \%$ difference. This is likely related to the lower order of the reconstructed fields which generates a different convergence point for FRT. Convergence in IRT is obtained rapidly with relatively small runtimes. Similarly to the single beam case, the convergence rate of FRT for energy conservation is significantly slower than using IRT. Once again, this is related to the noise in the reflected fields of the beams, which plays an important role in the 60 beam interaction since incident fields transfer a significant part of their energy to the reflected fields of adjacent beams.

Here it is also interesting to look at the spatial location where CBET is occurring. The CBET-induced permittivity perturbation $\delta \epsilon$ (see Eq. 17) is illustrated in Fig. 16, separated for the incident and reflected fields, as well as for the depletion and amplification parts. In this particular configuration, corresponding to the OMEGA laser in direct-drive, it appears that there is significant CBET gain at caustics. Notably, this is the case for the amplification component in the first sheet and the depletion component in the second sheet. This is a strong motivation for improving laser field modeling of caustics (removing associated tuning parameters), as is proposed in this paper through the Etalon Integral used in IFRIIT (see Sec. 2.3.

\subsection{Cryogenic implosion simulations}

We now present numerical tests results for ASTER+IFRIIT simulations of cryogenic ICF implosions. The present setup considered here corresponds to typical parameters for cryogenic direct-drive laser plasma experiments conducted on the OMEGA laser system [13]. 60 laser beams are focused at normal incidence on a cryogenic-temperature spherical shell of deuterated carbon (CD) and deuterium-tritum (DT) ice filled with DT gas (see Fig. 17 (a)). The laser pulses consist of a picket followed by a $1.5 \mathrm{~ns}$ drive, containing a total of $28 \mathrm{~kJ}$ of energy ${ }^{1}$ (see Fig. 17 (b)). Heating of the ablator material causes outward ejection of mass, which implodes the shell inward. As the shell implodes, its density and pressure increases, such that it eventually slows down at stagnation, and then disassembles. At stagnation, the density profile of the shell is modulated with perturbations corresponding to modes imposed by the

\footnotetext{
${ }^{1}$ The setup considered here corresponds to shot number 94712 conducted on the OMEGA laser system.
} 


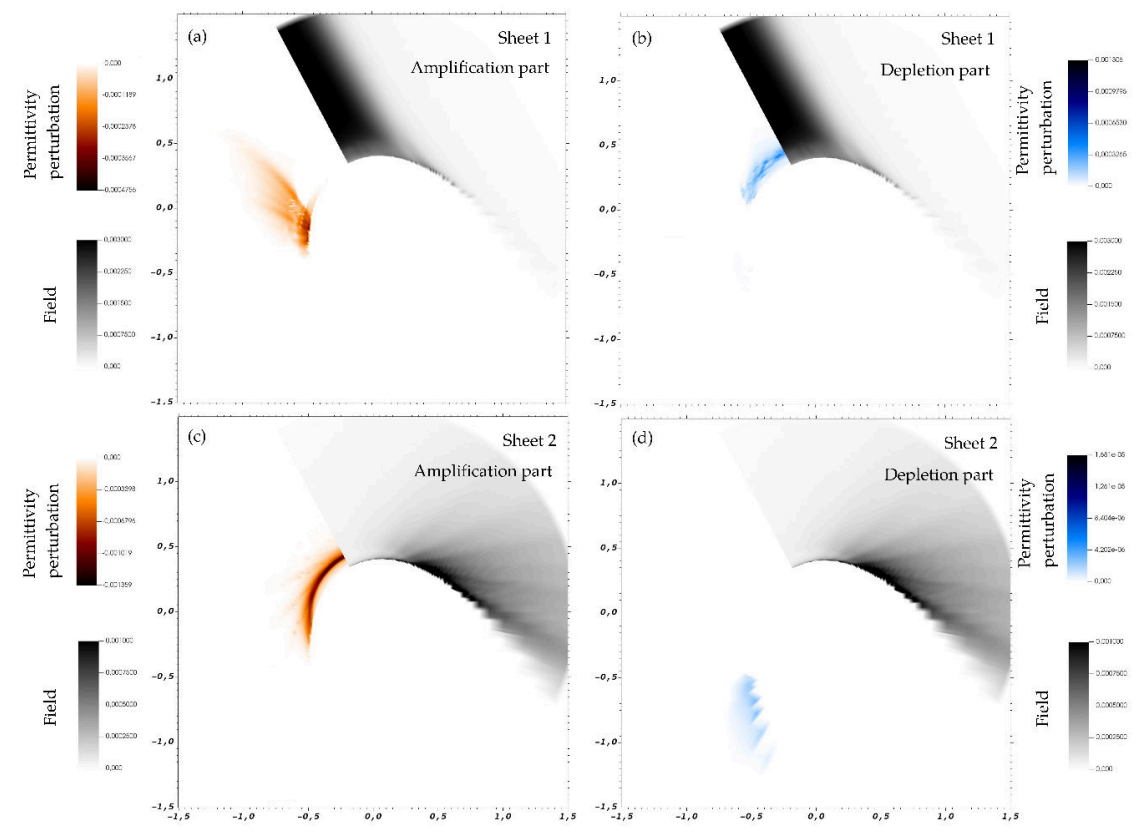

Figure 16: Typical position of CBET-induced permittivity perturbations in the 60-beams offline test case. Values shown are from a slice of OMEGA beam 10, incident from $\theta=58.852^{\circ}$ and $\phi=5.938^{\circ}$. Panels $(\mathrm{a}, \mathrm{b})$ give the contribution from the first sheet, and $(\mathrm{b}, \mathrm{c})$ from the second sheet. Negative permittivity perturbations $(\mathrm{a}, \mathrm{c})$ are shown in orange and represent field amplification. Positive permittivity perturbations $(\mathrm{b}, \mathrm{d})$ are shown in blue and represent field depletion. In all cases, the greyscale image represents the corresponding sheet field, in units of $v_{\text {osc }} / c$, and is represented to highlight the caustic location. In this particular setup, one can see that some part of the field amplification for sheet 1 and field depletion for sheet 2 occurs at caustics. 
laser drive. Long wavelength perturbations usually stem from the target chamber geometry, pointing errors, imbalance errors, and large-scale interaction processes such as CBET.

Cryogenic implosions are highly sensitive to modulations imprinted on targets at early time because they reach high convergence ratios. As such, any numerical perturbation present in the laser deposition scheme will get amplified by the implosion process and deform the capsule at stagnation, especially in the beginning of the coupling when the conduction zone is short. First, we present results of nominal calculations without any sources of asymmetry aside that of the target chamber in Sec. 5.4. While this is a calculation that requires 3-D geometries for the calculation of CBET, the illumination configuration with respect to the target is still symmetric. It is then useful to illustrate the code capability in the case of non-symmetric features. We consider the cases of target mis-alignment with respect to the Target Chamber Center (TCC) in Sec. 5.5, and that of beam power imbalance in Sec. 5.6.

In all cases, ASTER was used in a standard configuration for studies of laser-chamber-induced modes: the angular grid resolution was set to $128 \times 256$ cells in the polar and azimuthal directions, respectively. The radial directions consists of 750 cells with a varying resolution made to model the ablator, cryogenenic shell and gas and the respective interfaces, as well as the surrounding vacuum. The thermal transport was modeled using a Spitzer-Harm model with low temperature corrections [11], with a flux limitation of $15 \%$ for the picket pulse and no flux limitation afterwards.

\subsection{Baseline case}

\subsubsection{Target coupling and hotspot at convergence}

The absorption profile of the laser beams in the plasma is shown in Fig. 17 (b) alongside shell trajectories. IFRIIT predicts a loss of coupling due to CBET of $14.6 \%$, resulting in a delayed target stagnation and slightly lower convergence ratio. This results in a shift of the peak neutron production of $160 \mathrm{ps}$ and a yield over clean of $45 \%$ (see Figs. 18), whereby "clean" designates a case without CBET nor target offset or imbalance effects. The shape of the hotspot at stagnation is given in Fig. 17 (c, top row) for cases with and without CBET. The shell modulations seen in these figures can also be seen in the $\rho r$ metric, defined as the integral $\int_{0}^{\infty} \rho(r) \mathrm{d} r$ and shown in Fig. 19. Both the non-CBET and CBET cases show mostly regular and symmetric deformation patterns of the capsule density and $\rho r$ at stagnation. This implies that the level of numerical perturbations using IRT in IFRIIT is sufficiently low. The symmetry of the implosion in this ideal case is also illustrated in Fig. 20, showing the isocontours of density and DT gas/ice interface at stagnation in the case with CBET. It is also seen that mode $l=10$ is the dominant mode, which is imposed by the truncated icosahedron geometry of the OMEGA chamber. Here, CBET is seen to mainly amplify higher $l$ modes, creating small scale structures.

\subsubsection{Noise and mesh-induced modes}

Contrary to the relatively low noise level observed in the shell at stagnation in the IRT case, the FRT case shows significant losses of symmetry, as illustrated in Fig. 17 (c, bottom row). This can be attributed to mesh modes being imprinted significantly in the FRT case due to the lower order reconstruction method for the fields. Note that simulations using FRT with CBET were not conducted because the runtime was found to be a factor of 5 higher than IRT with CBET, consistent with the offline test cases presented in Sec. 5.2. In both the IRT and FRT runs, a spherical harmonics smoothing method was applied up to $1 \mathrm{~ns}$, filtering $l$-modes higher than 30 . This allows to filter small wavelength perturbations caused by numerical algorithms during the sensitive early time period. A comparison of the smoothing effect on the $\rho r$ maps at $1 \mathrm{~ns}$ in the IRT and FRT case is shown in Fig. 21(a,b). In that test case, the ideal solution would show no other modes than those imposed by the chamber, i.e. long scalelength modes. The figure illustrates how the IRT case is significantly smoother than FRT, even without spherical harmonics smoothing. Notably, the line-shaped structures seen in the FRT case without smoothing are related to the icosahedron mesh and stem from the subdivision of the sphere in 20 master tetrahedrons and the preferential octahedron splitting directions. The numerical noise caused by the master tetrahedrons interfaces can be partially mitigated using the alternated geodesic mesh configuration (see Sec. 4.1.2), especially in the FRT case, as illustrated in Fig. 21(c,d).

\subsection{Target offset error}

In the baseline case, we have considered an idealized beam setup of symmetric and balanced irradiation. In practice, pointing, beam balance and target alignment errors can compound to enhance the 3-D aspect of the configuration. Here we consider the case of a target mis-alignment with TCC. The upper range of target pointing errors reported in 
(a)

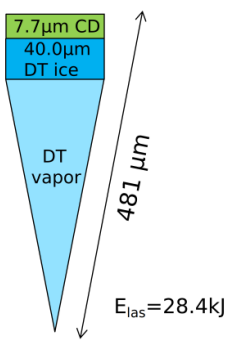

(b)

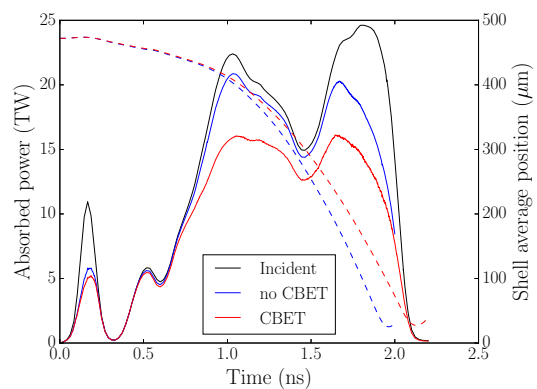

(c)

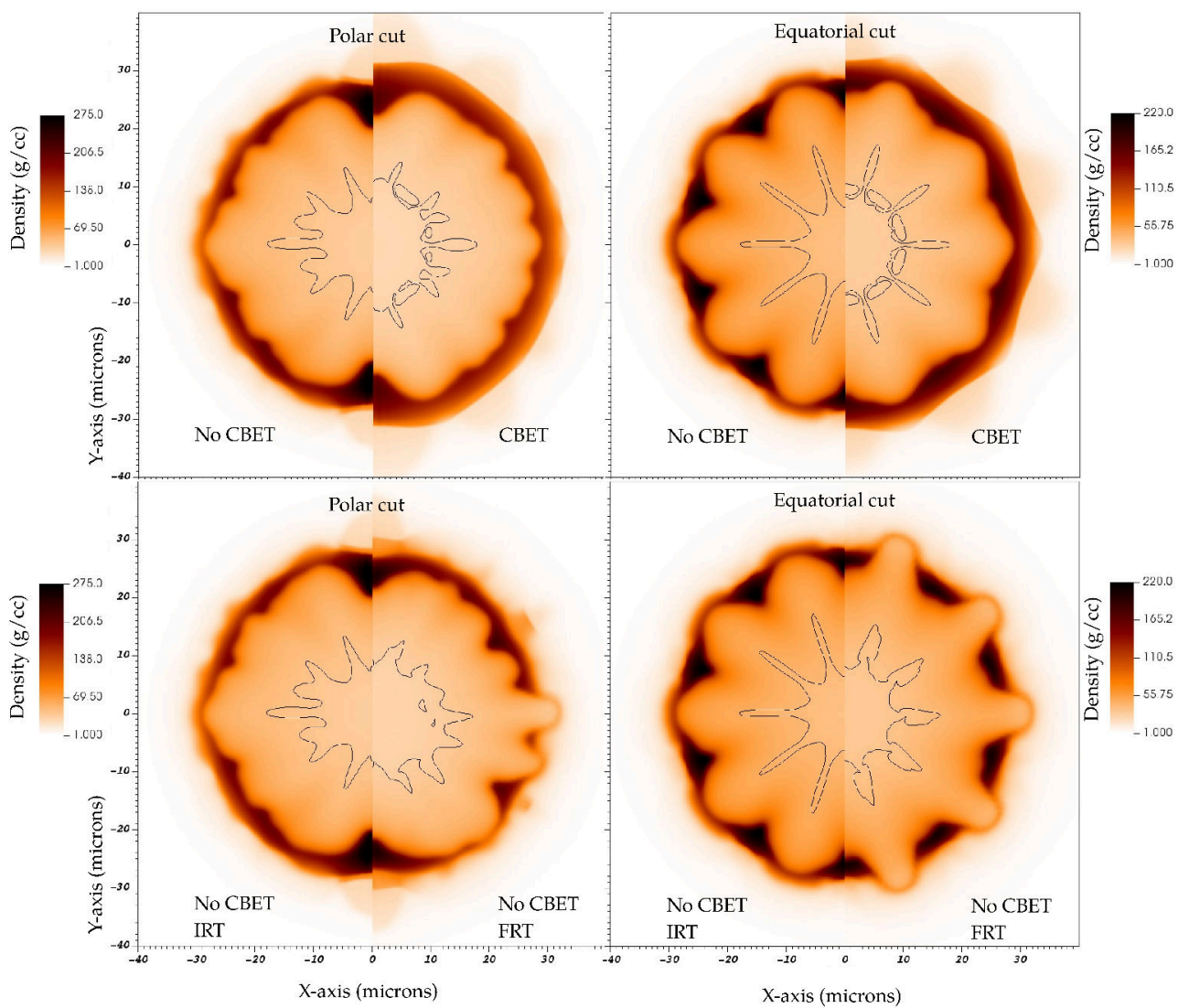

Figure 17: (a) Target configuration for the cryogenic implosion test case (facility shot \#94712). (b) Power metrics (solid lines) and shell trajectory (dashed lines) as a function of time. The incident laser power is shown in black, while results with and without CBET are shown in red and blue, respectively. (c) Polar (left row) and equatorial (right row) slices of density, comparing the effect of CBET (top) and the use of IRT versus FRT (bottom). Slices are taken close to the peak neutron rate, at $t=2.115 \mathrm{~ns}$ and $t=1.955 \mathrm{~ns}$ for the cases with and without CBET using IRT, respectively. In the case of FRT, the peak neutron rate is slightly shifted and the slice is taken at $t=1.94 \mathrm{~ns}$. In all cases, the solid line represents the DT ice/DT gas 50/50 volume of fluid contour. Polar slices are taken at $\theta=0$ and $\phi=0$ and equatorial slices at $\theta=90$ and $\phi=0$. Here, the choice of $\phi$ in the polar slices under-represents the maximum shell deformation amplitude (see Fig. 19). 
(a)

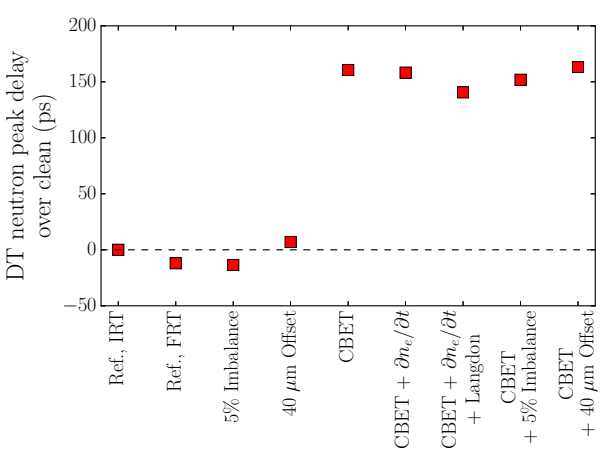

(b)

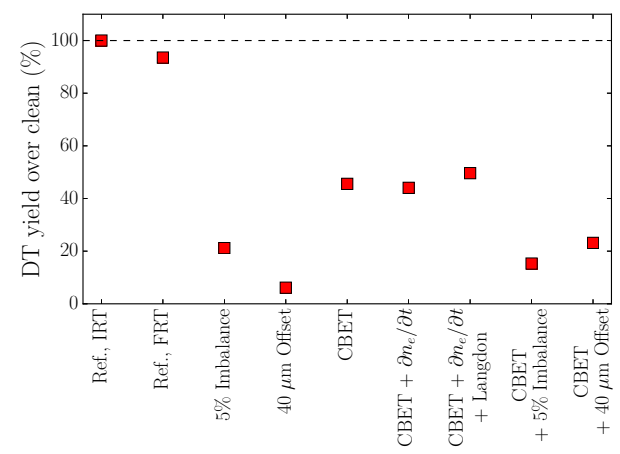

Figure 18: (a) Delay in the peak DT neutron production rate compared to the reference "clean" case, defined without CBET, offset nor beam imbalance. (b) DT yield over clean for the various cases. All simulations are conducted with IRT and include Langdon effect on absorption.
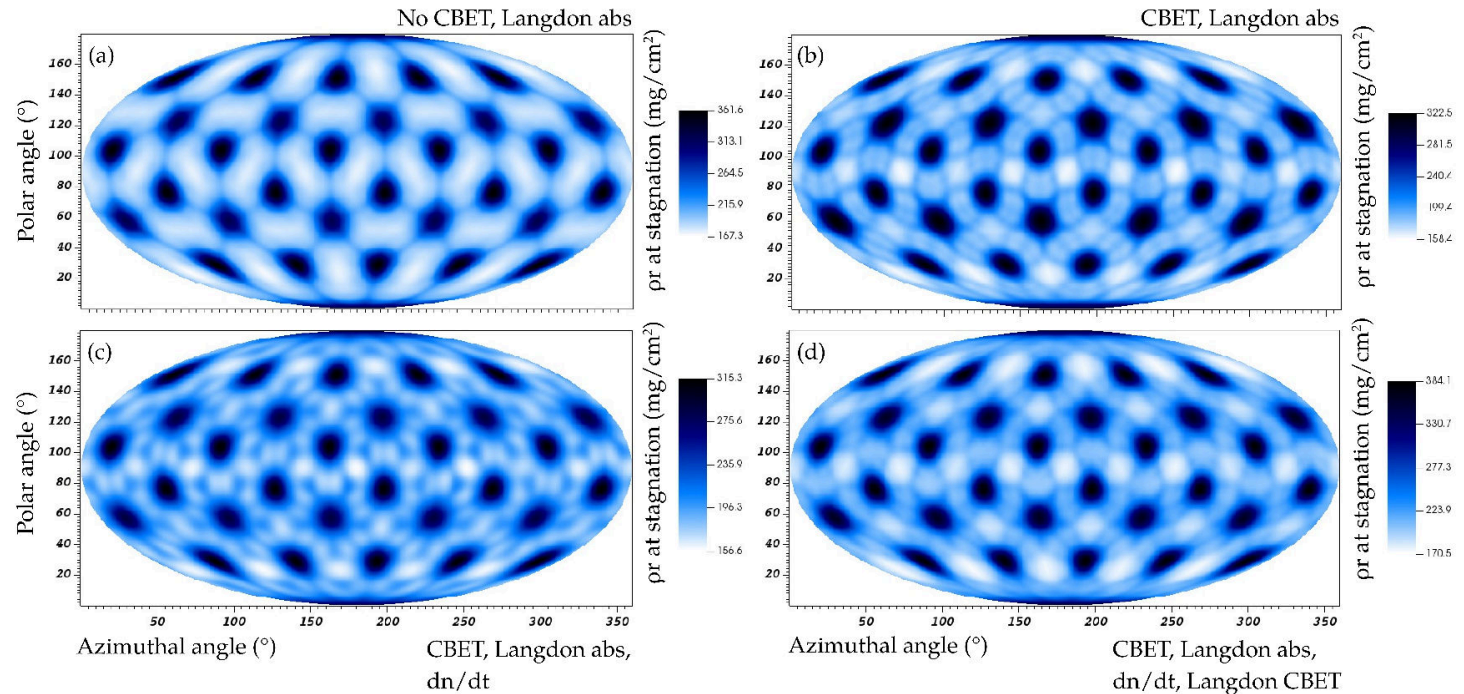

Figure 19: Mollweide projections of the target total $\rho r$ near stagnation for calculations using the IRT method. Simulations are shown without CBET (a) and with CBET (b,c,d). The frequency shift effect on CBET is added in (c,d), and the Langdon effect on CBET is added in (d). All cases include Langdon effect on absorption. Note that the values of $\rho r$ are not representative of the maximum $\rho r$ reached in each case, due to the time at which outputs are taken, which is not exactly that of stagnation. 
(a)

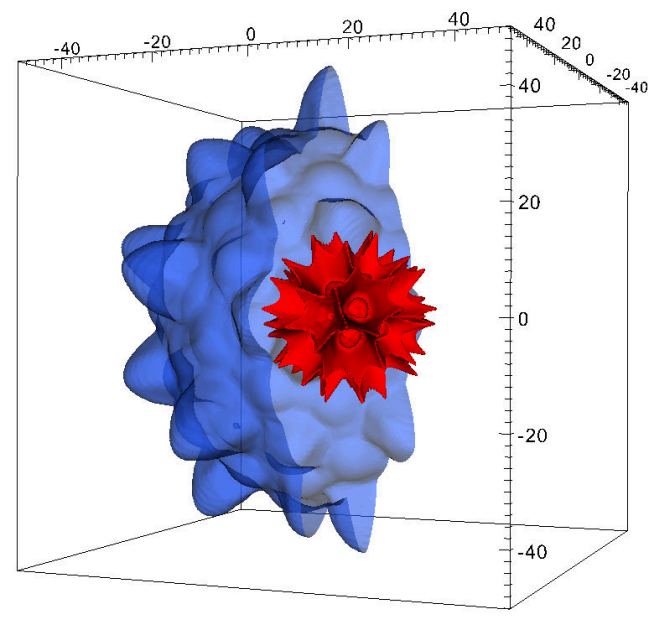

(b)

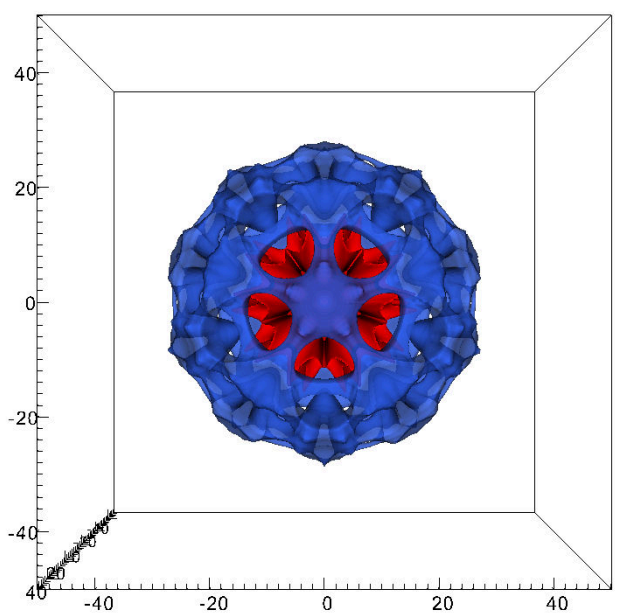

Figure 20: Cryogenic target at stagnation (at $2.115 \mathrm{~ns}$ ) in presence of CBET and in the ideal case (ideal pointing, balance and no target offset). In both panels, the 50/50 DT ice/gas VOF isocontour is shown in red. The blue field is the (a) $10 \mathrm{~g} / \mathrm{cc}$ and (b) $100 \mathrm{~g} / \mathrm{cc}$ density isocontour. In panel (a), the density isocontour is displayed in half in order to better show the hotspot contour. Axis ticks are in units of microns.
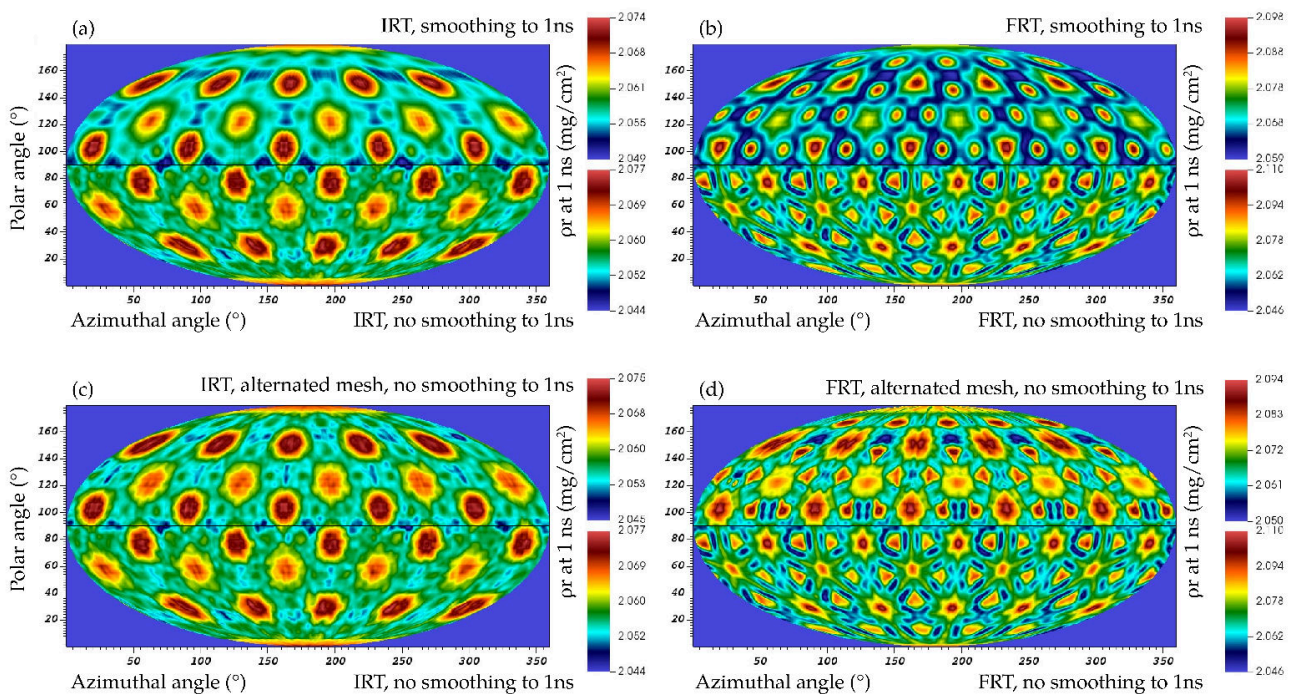

Figure 21: Mollweide projections of the target total $\rho r$ for calculations using IRT $(\mathrm{a}, \mathrm{c})$ and FRT $(\mathrm{b}, \mathrm{d})$ at $1 \mathrm{~ns}$. In the top rows of $(\mathrm{a}, \mathrm{c})$, the simulations use a spherical harmonics smoothing of the heat source term enabled up to $1 \mathrm{~ns}$. Computations use the hollow core and variable radial meshing for the geodesic raytracing mesh, except in the bottom rows of $(b, d)$ where the alternated geodesic mesh is used. The nonlinear colorbar is used to highlight small perturbations. Calculations are with Langdon effect on absorption and without CBET. 
(a)

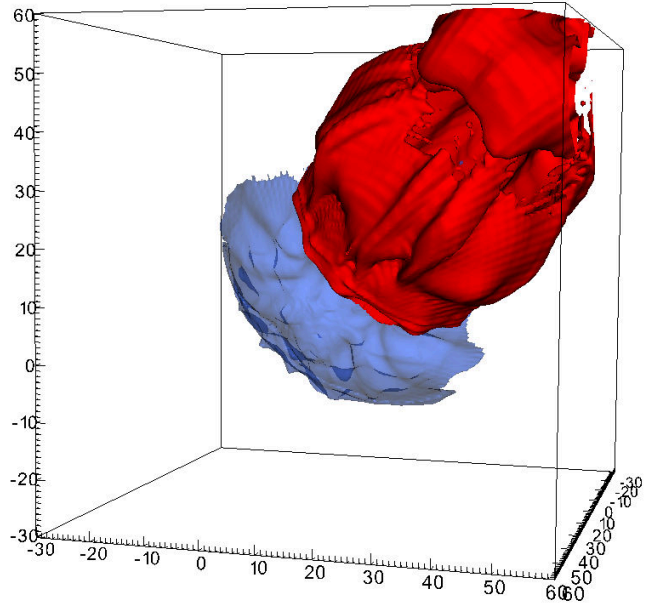

(c)

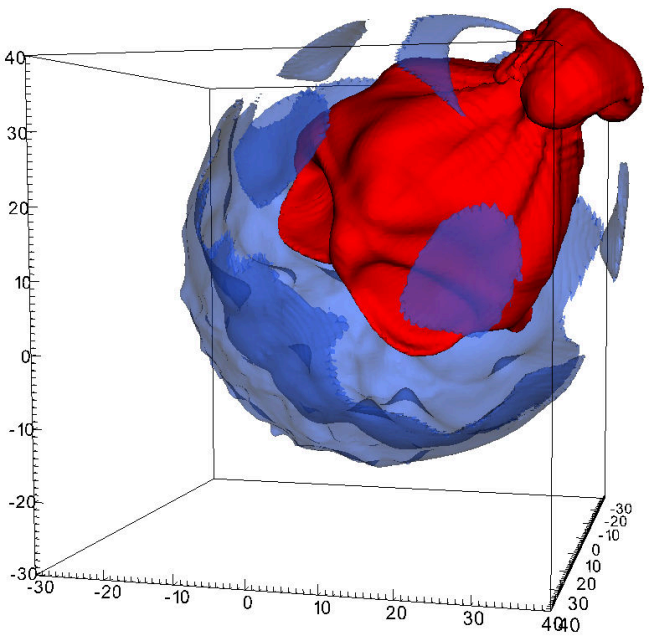

(b)

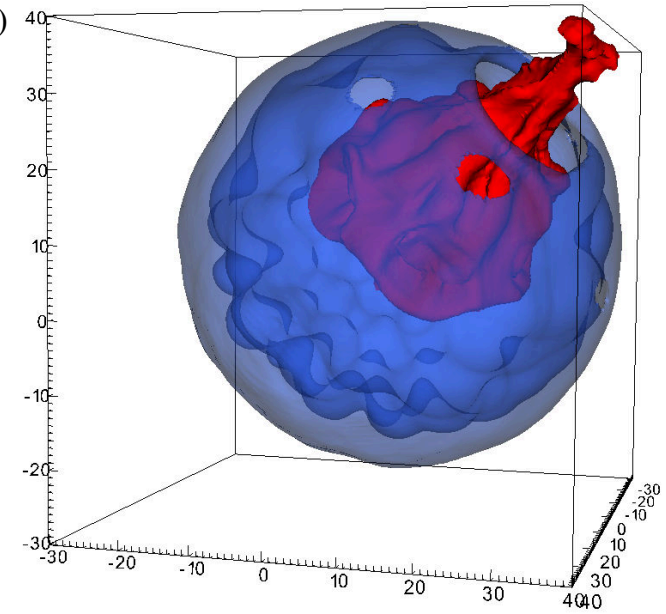

(d)

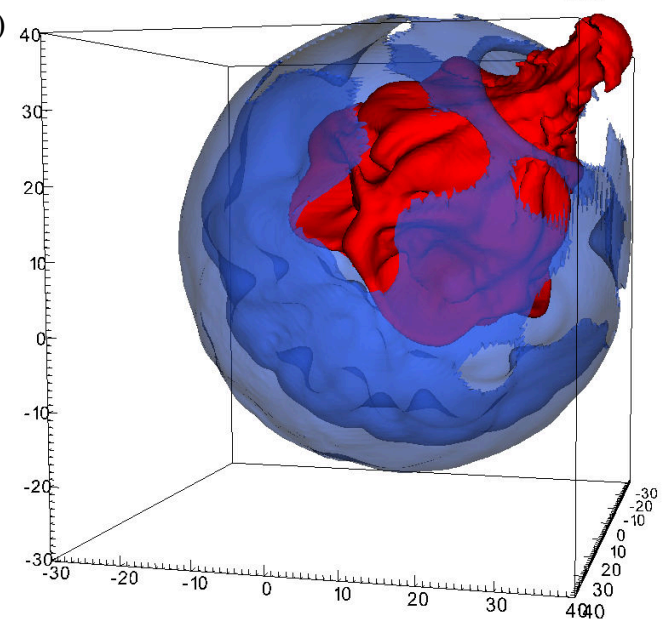

Figure 22: Cryogenic target at stagnation in presence of (a,b) a $40 \mu \mathrm{m}$ target offset in the $\theta=65, \phi=45$ direction, and (c,d) a $5 \%$ beam imbalance imposing a mode 1 in the same direction as the target offset. Run results without (a,c) and with (b,d) CBET enabled. In all figures, the $100 \mathrm{~g} / \mathrm{cc}$ density isocontour is shown in blue, the 50/50 DT ice/gas VOF isocontour is shown in red, and the axis ticks are in units of microns.

OMEGA experiments are offsets of $40 \mu \mathrm{m}$ with respect to TCC. Since both ASTER and IFRIIT use spherical grids which make assumption of spherical coordinates centered on 0 , the offset is instead modeled by shifting the incidence vectors of the beam in IFRIIT.

Asymmetries in the target at stagnation are illustrated in Fig. 22, and the resulting neutron yield and delays in Fig. 18. The target offset causes an imbalance in laser pressure through a spherical harmonics mode 1 in the heat deposition pattern. In the case with CBET, the shell at stagnation has been displaced by an additional $\sim 15 \mu \mathrm{m}$ in the offset direction, and is punctured with a jet of DT gas being pushed outside of the shell. Here CBET acts to mitigate the offset, by reducing laser pressure on the side opposed to the offset, where there is larger beam overlap and intensity. As such, when removing CBET, the target is exploded. The yield over clean for the offset cases is $23 \%$ with CBET and $6 \%$ without CBET.

\subsection{Beam imbalance case}

Similar to the target offset case, a source of 3-D asymmetry can be an imbalance in the laser power distribution. Here we consider an asymmetry in power balance of $5 \%$ standard deviation so as to produce a mode 1 in the illumination pattern. The resulting shell asymmetry at stagnation is similar to the target offset case, as reported in Figs. 22. In both cases with and without CBET, the shell has broken and a jet of DT gas is streaming from the capsule at 
(a)

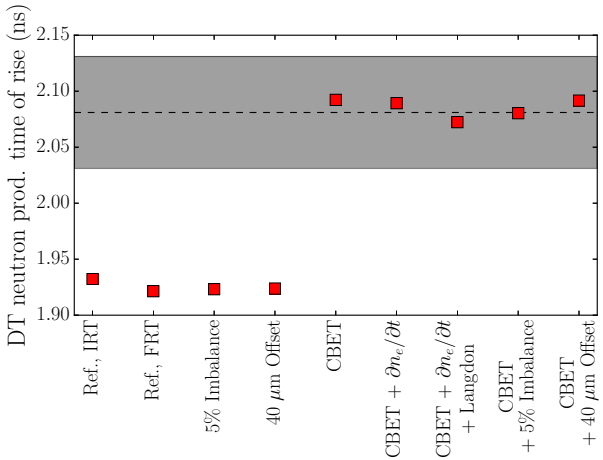

(b)

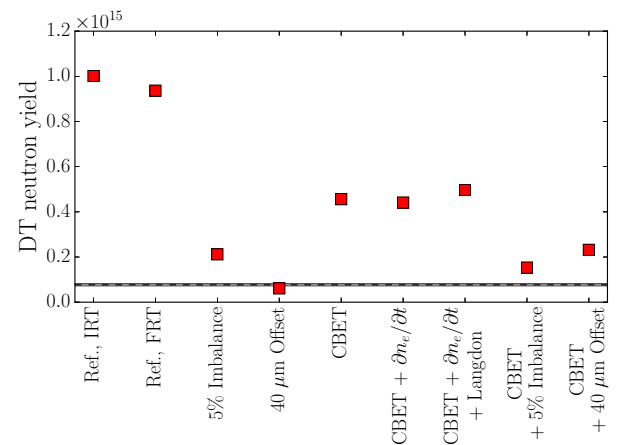

(c)

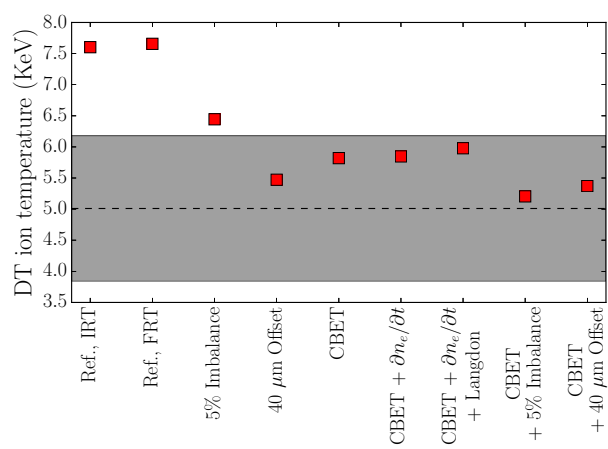

Figure 23: Comparison of neutron diagnostics data to simulations. In each panel, the measurements are shown with a dashed line for the average value and a grey shaded area for the error bar. (a) Time of rise of the DT neutron production, taken as the time where the production rate is $20 \%$ of the peak production. Data is from the NTD diagnostic. (b) Total DT neutron yield. The data is an average over 6 diagnostics (Cu Activation, H10 nTOF, $12 \mathrm{~m}$ nTOFn, 15.8m nTOF, 10.4m PD040, 15.9m MCP, PETAL nTOF). (c) Time-integrated average DT ion temperature. Results from the simulations are computed as $\left\langle T_{i}\right\rangle_{\mathrm{DT}}=\int T_{i, D T}(t) Y_{\mathrm{DT}}(t) \mathrm{d} t / \int Y_{\mathrm{DT}}(t) \mathrm{d} t$, where $T_{i, D T}(t)$ is the yield-weighted average (over space) ion temperature at time $t$. Data is taken from the same diagnostics as in panel (b). The measured DT ion temperature depends strongly on the measurement axis. As such, the data shown in (c) represents the range from the minimum to the maximum measured DT ion temperature.

stagnation. The decrease in yield due to the imbalance is not as severe as in the target offset case, with $20 \%$ yield over clean (see Fig. 18). In this particular case, CBET does not mitigate the imbalance as efficiently as the target offset, with $14.5 \%$ yield over clean with CBET and imbalance.

\subsection{Comparison to experimental data}

Numerical results are compared to experimental measurements obtained from the neutron diagnostics. These diagnostics measure the properties of DT produced neutrons in the hotspot to infer the time of target peak convergence (or bang time), the DT ion temperature and the DT neutron yield. The bang time is mostly sensitive to the accuracy of the laser-plasma coupling described in the simulation. Comparison with the data, shown in Fig. 23 (a), shows an excellent agreement with the simulations that include CBET. As illustrated in the simulation, beam imbalance and target offset has a weak effect on the bang time. The measured neutron yield and ion temperature are shown in Fig. $23(\mathrm{~b}, \mathrm{c})$. The comparisons to the simulation should here be considered with care. Unlike the bang time, which is a direct characteristic of the laser drive, the yield and ion temperatures are sensitive to many factors, which are not included in the current simulations or can be modeled wrongly, such as low modes, imprint, etc. In addition, ion temperature measurements strongly depend on the direction of measurements. Nonetheless, it is interesting to make the comparison with the various effects considered in the current setup. Here we can see that only simulations with CBET or with strong imbalance/target offset produce ion temperatures in the range of the measurement. However, most of the simulations strongly over-predict the DT yield, which is seen to be closest to the most degraded simulations with $40 \mu \mathrm{m}$ offset or CBET $+5 \%$ imbalance. While this does not imply that such effects were present during the shot, it illustrates the level of yield degradation still at play in those implosion experiments. 


\section{Conclusion}

The IFRIIT fluid-scale 3-D laser propagation code and its coupling to the ASTER 3-D radiative hydrodynamics code has been presented. IFRIIT combines several innovations in the algorithms employed, providing advantages in terms of numerical performances, accuracy, and physics models. Most of these novel features are enabled by the use of Inverse Ray Tracing (IRT), contrary to the conventional Forward Ray Tracing (FRT) used in laser propagation models in radiation hydrodynamics codes.

On the numerics side, the use of IRT permits to compute laser fields at order 1 in space (linear per mesh cell), contrary to FRT where laser parameters are binned in cells, at order 0 in space (constant per mesh cell). Furthermore, the use of IRT gives the opportunity to decorrelate the field reconstruction from the laser tracing. As such, in IFRIIT, fields are computed on an observation mesh that differs from the the raytracing mesh where rays propagate. This allows further optimization since higher mesh resolutions are required for ray trajectories than for field computations. This is especially noticeable when including non-linear effects such as Cross Beam Energy Transfer (CBET), which require significant MPI communications between beams. In addition, these two meshes are separated from the hydrodynamics mesh (of the ASTER code). This enables to adapt the laser and observation mesh grid resolution to the requirements of the laser propagation, independently from the hydrodynamics mesh. Furthermore, this enables to optimize the laser meshes: the lower memory footprint allows to use a domain duplication method, which is more efficient for ray tracing than the domain decomposition employed in hydrodynamics. Here we make use of a geodesic grid for the laser raytracing, which prevents introducing a potential artificial mode 2 in the ray field. Finally, IRT allows to speed up pump depletion iterations involved in CBET since the amplitude term of the ray parameters does not require to be recomputed. This is not the case in FRT where the field estimation step is tied in the amplitude computation step. On the downside, the use of IRT is limited to well-behaved permittivity profiles where the laser configuration is limited to two sheets and caustics remain of fold type. When treating more than 2 laser sheets, numerical errors compounds and prevent to accuratly reconstruct the second caustic or properly converge the inverse method on the third sheet. Similarly, concave permittivity profiles will lead to caustics of higher orders (e.g. cusp or swallowtail types) which may involve a large number of rays and hence be difficult to properly model. Another example is a target stalk, which surface would need to be accuratly meshed in order to employ the IRT algorithm, thus presenting a significant challenge. In all thoses cases, the ASTER/IFRIIT approach would fallback to use a standard FRT approach.

On the physics model side, IFRIIT also implements novel modeling capabilities. First, the use of IRT offers a natural separation of the incident and reflected fields. This separation is necessary when computing CBET, since it allows to compute self-amplification or self-depletion. This is often difficult to achieve in FRT-based model because it relies in a k-space separation which can loose precision near turning points. It is also ignored in current models dedicated to Indirect-Drive geometries, which implies that self-CBET is currently not modeled near holhraum walls in those models. Second, IRT allows to use the Etalon Integral method to reconstruct caustics fields from laser phase space parameters themselves. Caustics are often either ignored or treated with artificial numerical coefficients. In IFRIIT, the caustic calculation is self-consistent and does not use such free parameters. Finally, the state of the art of CBET modeling has been implemented in the code, namely: multi-sheeted energy transfer, Langdon effect on Ion Accoustic Wave response, frequency shift due to temporal variations of electron density, and polarization transport due to refraction - the latter not being described here.

Comparisons of IRT versus FRT performances have been conducted in a variety of numerical test cases involving CBET modeling. The significant advantages of IRT were highlighted: lower numerical noise, faster convergence rates for energy conservation, and significantly smaller runtimes. Additional tests were presented for inline calculations coupled to the hydrodynamics of ASTER in the case of cryogenic ICF implosions, which are highly sensitive to noise. It was demonstrated that ASTER+IFRIIT achieves efficient modeling of such experiments, with adequate runtimes and a low level of asymmetries induced by the numerical algorithms. Furthermore, the effects of frequency shift and Langdon effect on 3-D CBET were assessed, showing small changes in coupling and mode distributions. In addition to CBET, which is an inherently 3-D process, typical sources of 3-D asymmetries were explored: beam imbalance and target mis-alignment. An excellent agreement with the experiment was found in term of laser plasma coupling measured through bang-time. A good agreement is also found in term of DT ion temperature. However, the data shows significantly less DT yield than in the calculations, owing notably to effects not presently included in the simulations. 


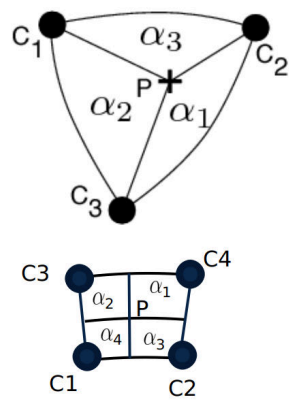

Figure B.24: Schematic representation of the spherical triangles and spherical quadrangles where interpolation is performed, in a given spherical layer. The $C_{i}$ represent either IFRIIT cell nodes or ASTER cell centers, and $P$ denotes the interpolation point.

The capacity to model 3-D hydrodynamics with 3-D laser propagation physics, including the effect of CBET, opens the way to many new studies. A non-exhaustive list of such possibilities are: the detailed effect of polarization in 3-D CBET, combined effects of target mis-pointing with beam imbalance and beam mis-pointing and non-cylindricallysymmetric laser irradiation configurations such as those used in Polar Direct Drive calculations or in experiments involving additional Thomson Scattering probe beams. In addition, since the IFRIIT mesh is non-structured and the observation mesh is arbitrary, it is in principle possible to modify the grids presented here for geometries departing significantly from quasi-spherical implosions. An application of interest would be to study the effects of 3-D engineering features such as target holders.

\section{Acknowledgments}

This work was granted access to the HPC resources of TGCC under the allocation 2020-A0070506129 and 2021A0090506129 made by GENCI. The software used in this work was developed in part at the University of Rochesters Laboratory for Laser Energetics.

\section{Appendix A. Inverse ray-tracing algorithm}

The algorithm used for resolving the IRT step is given in Alg. 1 and consists of two major steps. First, the 3-D location of laser caustics are determined. This allows to determine the sheet configuration. Each sheet is then mapped with probe rays sampled at fixed interval. The ensemble of points obtained in each sheet sampling is then triangulated to allow for interpolation of the probe rays parameters inside the sheets. Second, the minimization of the function $\left\|\mathbf{R}\left(\zeta_{1}, \zeta_{2}, \tau\right)-\mathbf{r}_{0}\right\|_{\infty}$ is conducted for each observation point $\mathbf{r}_{0}$ (R represents the raytracing function). Initialization of the multi-dimensional Newton solver is performed by interpolation of the probe ray parameters into the triangulated sheets.

\section{Appendix B. Interpolation methods}

When transferring data between the various grids, interpolation is required. Given a field $f$ that must be interpolated at point $\mathbf{P} \equiv\left\{r_{P}, \theta_{P}, \phi_{P}\right\}$, we first determine the spherical layers indices $k$ and $k+1$ such that $r_{k}<r_{P}<r_{k+1}$. Within each layer, the cell element that encloses $\mathbf{P}$ in the angular directions is found, and $f$ is angularly interpolated to $f\left(r_{k}, \theta_{P}, \phi_{P}\right)$ and $f\left(r_{k+1}, \theta_{P}, \phi_{P}\right)$. Since the interpolations are always from a polar grid, the cell elements in questions are either quadrangular or triangular when a pole is involved. The angular interpolation method is written as linear per spherical quadrangle or spherical triangle, such that the interpolated value of $f$ at $\left(\theta_{P}, \phi_{P}\right)$ reads: 


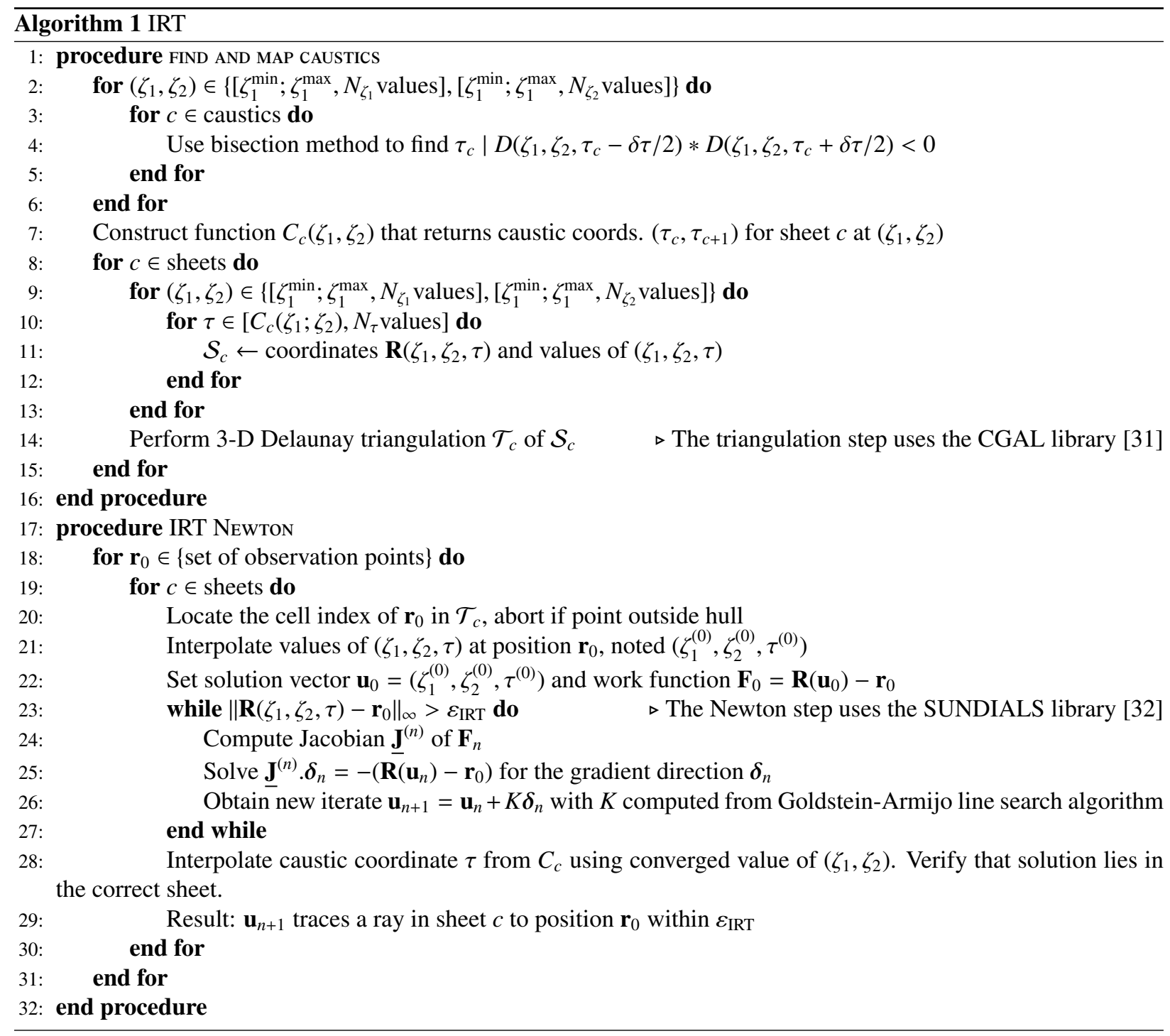




$$
\begin{array}{r}
w_{i}=\alpha_{i} / \sum_{j=1}^{N_{n}} \alpha_{j} \\
f\left(\theta_{P}, \phi_{P}\right)=\sum_{i=1}^{N_{n}} w_{i} f_{i}
\end{array}
$$

where $N_{n}$ is the number of nodes in the cell, $f_{i}$ is the value of $f$ at node $i$ and $w_{i}$ is a normalized weight. The weight associated with node $i$ is the spherical area $\alpha_{i}$ of the polygon formed by $P$ and the other nodes in the cell, normalized to the total spherical area of the cell. This is illustrated in Fig. B.24. The spherical areas for triangles are computed from the Cartesian node coordinates using L'Huillier's theorem, while the spherical area for the quadrangles is simply $r^{2} \sin \theta \delta \theta \delta \phi$ with $\delta \theta$ and $\delta \phi$ the appropriate angular lengths of the sub cells. The final value for $f(\mathbf{P})$ is obtained by linearly interpolating between the adjacent layers: $f(\mathbf{P})=(1-d) f\left(r_{k}, \theta_{P}, \phi_{P}\right)+d f\left(r_{k+1}, \theta_{P}, \phi_{P}\right)$ with $d=\left(r_{P}-r_{k}\right) /\left(r_{k+1}-r_{k}\right)$.

\section{Appendix C. Source term integration}

The squared field integral is performed in the ASTER cell using interpolated values at the 8 vertices, denoted $f_{j}$, $j \in[1,8]$. Given a cell defined between coordinates $\left\{r_{1}, r_{2}\right\},\left\{\theta_{1}, \theta_{2}\right\}$ and $\left\{\phi_{1}, \phi_{2}\right\}$, we write a simple bi-linear subgrid model for $f$ in layers $r_{1}$ and $r_{2}$ as $f\left(r_{m}, \theta, \phi\right)=A_{1 m}+A_{2 m} \theta+A_{3 m} \phi+A_{4 m} \theta \phi$, with $m \in[1,2]$. The $A_{k m}$ values are found by matching $f\left(r_{m}, \theta, \phi\right)$ with the interpolated values at the four nodes in the layer:

$$
\begin{array}{r}
A_{1 m}=\left[\left(f_{4 m} \phi_{1}-f_{3 m} \phi_{2}\right) \theta_{1}+\left(-f_{2 m} \phi_{1}+f_{1 m} \phi_{2}\right) \theta_{2}\right] /\left(\left(\phi_{1}-\phi_{2}\right)\left(\theta_{1}-\theta_{2}\right)\right) \\
A_{2 m}=\left[\left(f_{2 m}-f_{4 m}\right) \phi_{1}+\left(-f_{1 m}+f_{3 m}\right) \phi_{2}\right] /\left(\left(\phi_{1}-\phi_{2}\right)\left(\theta_{1}-\theta_{2}\right)\right) \\
A_{3 m}=\left[\left(f_{3 m}-f_{4 m}\right) \theta_{1}+\left(-f_{1 m}+f_{2 m}\right) \theta_{2}\right] /\left(\left(\phi_{1}-\phi_{2}\right)\left(\theta_{1}-\theta_{2}\right)\right) \\
A_{4 m}=\left[f_{1 m}-f_{3 m}-f_{2 m}+f_{4 m}\right] /\left(\left(\phi_{1}-\phi_{2}\right)\left(\theta_{1}-\theta_{2}\right)\right),
\end{array}
$$

with $f\left(r_{m}, \theta_{1}, \phi_{1}\right) \equiv f_{1 m}, f\left(r_{m}, \theta_{1}, \phi_{2}\right) \equiv f_{2 m}, f\left(r_{m}, \theta_{2}, \phi_{1}\right) \equiv f_{3 m}$ and $f\left(r_{m}, \theta_{2}, \phi_{2}\right) \equiv f_{4 m}$. The function $f\left(r_{m}, \theta, \phi\right)$ is then integrated over the cell surface at $r_{m}$ :

$$
\begin{array}{r}
F\left(r_{m}\right)=\int_{\theta 1}^{\theta_{2}} \int_{\phi_{1}}^{\phi_{2}} f\left(r_{m}, \theta, \phi\right) \sin \theta \mathrm{d} \theta \mathrm{d} \phi= \\
\left(( \phi _ { 1 } - \phi _ { 2 } ) \left(-\left(\left(2 A_{1 m}+2 A_{2 m} \theta_{1}+\left(\phi_{1}+\phi_{2}\right)\left(A_{3 m}+A_{4 m} \theta_{1}\right)\right) \cos \left(\theta_{1}\right)\right)+\right.\right. \\
\left(2 A_{1 m}+2 A_{2 m} \theta_{2}+\left(\phi_{1}+\phi_{2}\right)\left(A_{3 m}+A_{4 m} \theta_{2}\right)\right) \cos \left(\theta_{2}\right)+ \\
\left.\left.\left(2 A_{2 m}+A_{4 m}\left(\phi_{1}+\phi_{2}\right)\right)\left(\sin \left(\theta_{1}\right)-\sin \left(\theta_{2}\right)\right)\right)\right) / 2 .
\end{array}
$$

The volume integral is finally obtained by assuming a linear form for $F(r)$ and integrating over $r^{2} \mathrm{~d} r$ :

$$
\begin{array}{r}
\int_{V_{i}} f(r, \theta, \phi) \mathrm{d} V=\int_{r_{1}}^{r_{2}} r^{2}\left[F\left(r_{1}\right)+\left(F\left(r_{2}\right)-F\left(r_{1}\right)\right)\left(r-r_{1}\right) /\left(r_{2}-r_{1}\right)\right] \mathrm{d} r \\
=\left(\left(r_{2}-r_{1}\right)\left(\left(3 F\left(r_{1}\right)+F\left(r_{2}\right)\right) r_{1}^{2}+2\left(F\left(r_{1}\right)+F\left(r_{2}\right)\right) r_{1} r_{2}+\left(F\left(r_{1}\right)+3 F\left(r_{2}\right)\right) r_{2}^{2}\right)\right) / 12 .
\end{array}
$$

The simple projection method presented here only conserves energy when there are many ASTER cells per IFRIIT cell. While this is the intended behavior since ASTER uses much finer resolutions in general, this is not always the case because of the variable meshing in IFRIIT and the many specific coronal plasma configuration that can occur. While this could be addressed using a different projection method, cases where ASTER cells are larger than IFRIIT cells simply mean that the hydrodynamic mesh resolution is insufficient to capture some fine details of the field structure. We choose to leave this behavior, as one can always increase the hydrodynamic mesh resolution to compensate. As such, the heat source term of each beam is simply renormalized after projection to the intended energy deposition from IFRIIT. 
[1] R. Betti and O. A. Hurricane. Inertial-confinement fusion with lasers. Nature Physics, 12:435-448, May 2016.

[2] R. S. Craxton, K. S. Anderson, T. R. Boehly, V. N. Goncharov, D. R. Harding, J. P. Knauer, R. L. McCrory, P. W. McKenty, D. D. Meyerhofer, J. F. Myatt, A. J. Schmitt, J. D. Sethian, R. W. Short, S. Skupsky, W. Theobald, W. L. Kruer, K. Tanaka, R. Betti, T. J. B. Collins, J. A. Delettrez, S. X. Hu, J. A. Marozas, A. V. Maximov, D. T. Michel, P. B. Radha, S. P. Regan, T. C. Sangster, W. Seka, A. A. Solodov, J. M. Soures, C. Stoeckl, and J. D. Zuegel. Direct-drive inertial confinement fusion: A review. Physics of Plasmas, 22(11):110501, 2015.

[3] John D. Lindl, Peter Amendt, Richard L. Berger, S. Gail Glendinning, Siegfried H. Glenzer, Steven W. Haan, Robert L. Kauffman, Otto L. Landen, and Laurence J. Suter. The physics basis for ignition using indirect-drive targets on the national ignition facility. Physics of Plasmas, 11(2):339-491, 2004.

[4] S. A. Slutz, W. A. Stygar, M. R. Gomez, K. J. Peterson, A. B. Sefkow, D. B. Sinars, R. A. Vesey, E. M. Campbell, and R. Betti. Scaling magnetized liner inertial fusion on $\mathrm{z}$ and future pulsed-power accelerators. Physics of Plasmas, 23(2):022702, 2016.

[5] W. L. Kruer. The Physics of Laser Plasma Interactions. Westview Press, University of California, Los Angeles, 2003.

[6] D. Batani, S. Baton, A. Casner, S. Depierreux, M. Hohenberger, O. Klimo, M. Koenig, C. Labaune, X. Ribeyre, C. Rousseaux, G. Schurtz, W. Theobald, and V.T. Tikhonchuk. Physics issues for shock ignition. Nuclear Fusion, 54(5):054009, 2014.

[7] T. B. Kaiser. "laser ray tracing and power deposition on an unstructured three-dimensional grid". Phys. Rev. E, 61:895, January 2000.

[8] D. J. Strozzi, E. A. Williams, D. E. Hinkel, D. H. Froula, R. A. London, and D. A. Callahan. Ray-based calculations of backscatter in laser fusion targets. Physics of Plasmas, 15(10), 2008.

[9] P. Michel, L. Divol, E. A. Williams, C. A. Thomas, D. A. Callahan, S. Weber, S. W. Haan, J. D. Salmonson, N. B. Meezan, O. L. Landen, S. Dixit, D. E. Hinkel, M. J. Edwards, B. J. MacGowan, J. D. Lindl, S. H. Glenzer, and L. J. Suter. Energy transfer between laser beams crossing in ignition hohlraums. Physics of Plasmas, 16(4), 2009.

[10] D. J. Strozzi, D. S. Bailey, P. Michel, L. Divol, S. M. Sepke, G. D. Kerbel, C. A. Thomas, J. E. Ralph, J. D. Moody, and M. B. Schneider. Interplay of laser-plasma interactions and inertial fusion hydrodynamics. Phys. Rev. Lett., 118:025002, Jan 2017.

[11] I. V. Igumenshchev, V. N. Goncharov, F. J. Marshall, J. P. Knauer, E. M. Campbell, C. J. Forrest, D. H. Froula, V. Yu. Glebov, R. L. McCrory, S. P. Regan, T. C. Sangster, S. Skupsky, and C. Stoeckl. Three-dimensional modeling of direct-drive cryogenic implosions on omega. Physics of Plasmas, 23(5):052702, 2016.

[12] I. V. Igumenshchev, D. T. Michel, R. C. Shah, E. M. Campbell, R. Epstein, C. J. Forrest, V. Yu. Glebov, V. N. Goncharov, J. P. Knauer, F. J. Marshall, R. L. McCrory, S. P. Regan, T. C. Sangster, C. Stoeckl, A. J. Schmitt, and S. Obenschain. Three-dimensional hydrodynamic simulations of omega implosions. Physics of Plasmas, 24(5):056307, 2017.

[13] T.R Boehly, D.L Brown, R.S Craxton, R.L Keck, J.P Knauer, J.H Kelly, T.J Kessler, S.A Kumpan, S.J Loucks, S.A Letzring, F.J Marshall, R.L McCrory, S.F.B Morse, W Seka, J.M Soures, and C.P Verdon. Initial performance results of the OMEGA laser system. Optics Communications, 133(1-6):495 - 506, 1997.

[14] Y. A. Kravtsov and N. Y. Zhu. Theory of Diffraction, Heuristic Approaches. Alpha Science Series on Wave Phenomena. Alpha Science International LTD., Oxford, U.K., 2010.

[15] A. Colaïtis, J. P. Palastro, R. K. Follett, I. V. Igumenschev, and V. Goncharov. Real and complex valued geometrical optics inverse ray-tracing for inline field calculations. Physics of Plasmas, 26(3):032301, 2019

[16] R. K. Follett, J. G. Shaw, J. F. Myatt, V. N. Goncharov, D. H. Edgell, D. H. Froula, and J. P. Palastro. Ray-based modeling of cross-beam energy transfer at caustics. Phys. Rev. E, 98:043202, Oct 2018.

[17] A. Bruce Langdon. Nonlinear inverse bremsstrahlung and heated-electron distributions. Phys. Rev. Lett., 44:575-579, Mar 1980.

[18] D. Turnbull, A. Colaïtis, A. M. Hansen, A. L. Milder, J. P. Palastro, J. Katz, C. Dorrer, B. E. Kruschwitz, D. J. Strozzi 2, and D. H. Froula. Impact of the langdon effect on crossed-beam energy transfer. Nature Physics, 16:181-185, February 2020. Nature Physics.

[19] A. Colaïtis, R. K. Follett, J. P. Palastro, I. Igumenschev, and V. Goncharov. Adaptive inverse ray-tracing for accurate and efficient modeling of cross beam energy transfer in hydrodynamics simulations. Physics of Plasmas, 26(7):072706, 2019.

[20] A. Colaïtis, T. Chapman, D. Strozzi, L. Divol, and P. Michel. A tesselation-based model for intensity estimation and laser plasma interactions calculations in three dimensions. Physics of Plasmas, 25(3):033114, 2018.

[21] T. Dewandre, J. R. Albritton, and E. A. Williams. Doppler shift of laser light reflected from expanding plasmas. Physics of Fluids, 24:528536, March 1981.

[22] Y. A. Kravtsov and P. Berczynski. "gaussian beams in inhomogeneous media: A review". Studia Geophysica et Geodaetica, 51:1-36, January 2007.

[23] A. Colaïtis, G. Duchateau, P. Nicolaï, and V. Tikhonchuk. Towards modeling of nonlinear laser-plasma interactions with hydrocodes: The thick-ray approach. Phys. Rev. E, 89:033101, March 2014.

[24] J. A. Marozas, M. Hohenberger, M. J. Rosenberg, D. Turnbull, T. J. B. Collins, P. B. Radha, P. W. McKenty, J. D. Zuegel, F. J. Marshall, S. P. Regan, T. C. Sangster, W. Seka, E. M. Campbell, V. N. Goncharov, M. W. Bowers, J.-M. G. Di Nicola, G. Erbert, B. J. MacGowan, L. J. Pelz, J. Moody, and S. T. Yang. Wavelength-detuning cross-beam energy transfer mitigation scheme for direct drive: Modeling and evidence from national ignition facility implosions. Physics of Plasmas, 25(5):056314, 2018.

[25] J. Delettrez, R. Epstein, M. C. Richardson, P. A. Jaanimagi, and B. L. Henke. Effect of laser illumination nonuniformity on the analysis of time-resolved x-ray measurements in uv spherical transport experiments. Phys. Rev. A, 36:3926-3934, Oct 1987.

[26] D. H. Edgell, R. K. Follett, I. V. Igumenshchev, J. F. Myatt, J. G. Shaw, and D. H. Froula. Mitigation of cross-beam energy transfer in symmetric implosions on omega using wavelength detuning. Physics of Plasmas, 24(6):062706, 2017.

[27] R. K. Follett, D. H. Edgell, D. H. Froula, V. N. Goncharov, I. V. Igumenshchev, J. G. Shaw, and J. F. Myatt. Full-wave and ray-based modeling of cross-beam energy transfer between laser beams with distributed phase plates and polarization smoothing. Physics of Plasmas, 24(10):103128, 2017.

[28] V. M. Babic and V. S. Buldyrev. Short-Wavelength Diffraction Theory: Asymptotic Methods. Springer Series on Wave Phenomena. SpringerVerlag, 1972.

[29] R. Thom. Structural stability and morphogenesis. CRC Press, 1975.

[30] D. Pesme, G. Bonnaud, M. Casanova, R. Dautray, C. Labaune, G. Laval, L. Bergé, and J.P. Watteau. La fusion thermonucléaire inertielle par laser : l'interaction laser-matière part. 1, vol. 1. (French Edition). Synthèses. Eyrolles, 61, Boulevard Saint-Germain, 1993. 
[31] The CGAL Project. CGAL User and Reference Manual. CGAL Editorial Board, 5.1 edition, 2020.

[32] Alan C Hindmarsh, Peter N Brown, Keith E Grant, Steven L Lee, Radu Serban, Dan E Shumaker, and Carol S Woodward. SUNDIALS: Suite of nonlinear and differential/algebraic equation solvers. ACM Transactions on Mathematical Software (TOMS), 31(3):363-396, 2005. 